\title{
Binary Asteroid Observation Orbits from a Global Dynamical Perspective*
}

\author{
F. Gabern ${ }^{\dagger \ddagger}$, W. S. Koon ${ }^{\dagger}$, J. E. Marsden ${ }^{\dagger}$, and D. J. Scheeres ${ }^{\S}$
}

Abstract. We study spacecraft motion near a binary asteroid by means of theoretical and computational tools from geometric mechanics and dynamical systems. We model the system assuming that one of the asteroids is a rigid body (ellipsoid) and the other a sphere. In particular, we are interested in finding periodic and quasi-periodic orbits for the spacecraft near the asteroid pair that are suitable for observations and measurements. First, using reduction theory, we study the full two body problem (gravitational interaction between the ellipsoid and the sphere) and use the energy-momentum method to prove nonlinear stability of certain relative equilibria. This study allows us to construct the restricted full three-body problem (RF3BP) for the spacecraft motion around the binary, assuming that the asteroid pair is in relative equilibrium. Then, we compute the modified Lagrangian fixed points and study their spectral stability. The fixed points of the restricted three-body problem are modified in the RF3BP because one of the primaries is a rigid body and not a point mass. A systematic study depending on the parameters of the problem is performed in an effort to understand the rigid body effects on the Lagrangian stability regions. Finally, using frequency analysis, we study the global dynamics near these modified Lagrangian points. From this global picture, we are able to identify (almost-) invariant tori in the stability region near the modified Lagrangian points. Quasi-periodic trajectories on these invariant tori are potentially convenient places to park the spacecraft while it is observing the asteroid pair.

Key words. asteroid pairs, spacecraft dynamics, stability, frequency analysis, invariant tori, quasi-periodic motion

AMS subject classifications. 37N05, 70F07, 70F 15

DOI. $10.1137 / 050641843$

1. Introduction. The dynamics of asteroid pairs has recently become a topic of interest, specially since the first binary asteroid, Ida-Dactyl, was discovered by the Galileo spacecraft in 1993. It is currently estimated that up to $20 \%$ of near-earth asteroids (NEA) are binaries [26], and other examples have been found among the asteroid main-belt, in the Trojan swarms and as transneptunian objects [33]. The problem of two rigid bodies orbiting around each other

* Received by the editors October 4, 2005; accepted for publication (in revised form) by T. Kaper February 14, 2006; published electronically June 2, 2006. This work was partly supported by the California Institute of Technology President's Fund, NSF-ITR grant ACl-0204932, NSF grant DMS-0505711, and by ICB, the Institute for Collaborative Biotechnologies, through ARO grant DAAD19-03-D-0004.

http://www.siam.org/journals/siads/5-2/64184.html

${ }^{\dagger}$ Control and Dynamical Systems, California Institute of Technology, 07-81, Pasadena, CA 91125 (gabern@ cds.caltech.edu, koon@cds.caltech.edu, marsden@cds.caltech.edu). The first author was supported by the 2004 Fulbright-Generalitat de Catalunya postdoctoral program, the MCyT/FEDER grant BFM2003-07521-C02-01, and the CIRIT grant 2001SGR-70.

${ }^{\ddagger}$ Current address: Departament de Matemàtica Aplicada I, Universitat Politècnica de Catalunya, Diagonal 647, 08028 Barcelona, Spain (frederic.gabern@upc.edu).

${ }^{\S}$ Department of Aerospace Engineering, University of Michigan, Ann Arbor, MI 48109-2140 (scheeres@umich. edu). This author acknowledges support from the Jet Propulsion Laboratory/California Institute of Technology through a grant from the Director's Research Discretionary Fund. 
attracted by their mutual gravitational forces is challenging from the geometric mechanics point of view [5]. Moreover, a very interesting problem with applications to astrodynamics is the description of the dynamics of a massless particle (e.g., a spacecraft) moving under the influence of the binary $[41,11]$. Indeed, binaries can be used as real-life laboratories to test rigid-body gravitational dynamics. As such, these theoretical studies may be relevant for a possible future mission to binaries.

The objective of this paper is to construct and study a model for the motion of a satellite orbiting a binary asteroid. To do this, we first have to develop a model for the asteroid pair itself. A binary asteroid provides a canonical model for general full body problems (FBPs); see $[24,39]$. FBPs are concerned with the dynamical interaction between distributed bodies of finite mass. In particular, the full two-body problem (F2BP) considers the dynamics of two spatially extended bodies that interact via their mutual gravitational fields. In this paper, we consider the "sphere restriction" of the F2BP [20]. That is, it is assumed that one of the rigid bodies is spherically symmetric and thus can be considered as a point mass. As for the other rigid body, we assume that it is a triaxial ellipsoid. The study of this simple model of an asteroid pair will give some hints on the dynamics of spacecrafts about binaries, and it can be generalized further using, for instance, more complicated potentials [47].

One of our first goals is to find stable relative equilibria of this F2BP with the property that we can later build models for the satellite motion around the pair. Moreover, as we look first for simple relative equilibria, we also assume that the two bodies are restricted to moving in a plane. This makes the reduction process and the equilibria characterization much simpler, but not too simple, as the coupling between rotational and translational motion is still there. For a probe sent to a binary asteroid, it is plausible to assume that the underlying F2BP is in some type of relative equilibrium. As the study of more complex models will be important in the future, we have to first understand the simplified cases. Thus, we devote the first part of the paper to studying the F2BP by means of reduction methods [28], to identifying its relative equilibria, and to proving nonlinear stability for some cases with the aid of the energy-momentum method [44].

Following this, we choose a particular stable relative equilibria of the F2BP, which corresponds to a periodic orbit in the original system, to construct a model for the satellite orbiting the binary. This model is a restricted problem of three bodies, but one of the primaries is a rigid body. In the literature this type of model has been called the restricted full three-body problem or RF3BP [41].

As is well known [45], the restricted three-body problem (RTBP) has five equilibrium points, and two of them form an equilateral triangle with the primaries. These equilateral equilibrium points, also called Lagrangian points, may persist when one of the primaries is not a point mass but a distributed body $[2,41,12]$. The position and stability properties of these points are, of course, affected by the perturbation and are thus modified. In the second part of the paper, we study how these equilibrium points are modified depending on the variation of the parameters of the problem.

In some previous works $[11,12]$, we have studied this problem using normal form techniques near the triangular points of some particular RF3BP, which are simpler than the ones presented here. Even though these tools give quite satisfactory results for a range of parameters, the zone around the fixed points where the dynamics can be described by the normal 
form is not very big. In this paper, we extend the description of this dynamics to larger regions of the phase space.

To achieve this goal, in the last part of the paper we apply a dynamical systems-based tool, namely frequency map analysis $[21,37,16]$, to study the global dynamics around the Lagrangian points. We are able to identify relatively large neighborhoods of these equilibrium points in phase space at which the trajectories are stable for a long time. As far as we know, this is the first time that this powerful numerical tool (frequency analysis) has been applied to orbit mechanics about asteroids.

Previously, a wavelet-based frequency analysis [46] has been used to study the transport in the Sun-Jupiter RTBP. The advantage of this method appears in problems where the frequencies vary with time, for example when there is relatively "fast" transport from one region of phase space to another. In our problem, we are interested in the dynamics close to an elliptic point, and the results given by the wavelet method should be similar to those presented here. In particular, we look for tori that are "invariant enough," i.e., tori where we can place the spacecraft for a long enough time (the meaning of this will be clarified later on). In this case, if there is transport between nearby tori, the transport should be slow.

The paper is organized as follows. In section 2, we develop and study a model of the asteroid pair. The relative equilibria of the reduced binary system are characterized, and their stability is studied by means of the energy-momentum method. In section 3 , we construct the model for the satellite orbiting the asteroid pair, i.e., the RF3BP, and we find the new coordinates of the modified Lagrangian points of the RTBP and study its spectral stability. In section 4, we study the global dynamics near the Lagrangian points of the RF3BP by means of frequency analysis methods. This global study allows us to find (almost-) invariant tori and trajectories very close to quasi-periodic, which are pretty suitable for the satellite. Finally, in section 5, our conclusions and future directions are presented. For the convenience of the reader, we add in the appendix a brief review on Abelian reduction theory, which is used in section 2 to study the F2BP.

\section{Stability of the asteroid pair.}

2.1. Reduced model for the binary. To model the asteroid pair, we consider the mechanical system of a triaxial ellipsoid and a sphere that interact via the mutual gravitational potential and are allowed to move in a plane. In an inertial reference frame, the kinetic energy of the system is

$$
K=\frac{1}{2} m\|\dot{\mathbf{q}}\|^{2}+\frac{1}{2} M\|\dot{\mathbf{Q}}\|^{2}+\frac{1}{2} I_{z z} \dot{\varphi}^{2}
$$

where $\mathbf{q}$ and $\mathbf{Q}$ are the positions of the sphere's center and the barycenter of the ellipsoid and $m$ and $M$ are, respectively, the masses of the sphere and the ellipsoid. $I_{z z}$ is the component orthogonal to the plane of motion of the inertia tensor of the ellipsoid, and the angle $\varphi$ is as shown in Figure 1.

This system is invariant under translations. Thus, defining the relative position of the bodies as $\mathbf{r}=\mathbf{q}-\mathbf{Q}$ and taking as unit of mass the reduced mass, i.e., $m M /(m+M)=1$, 


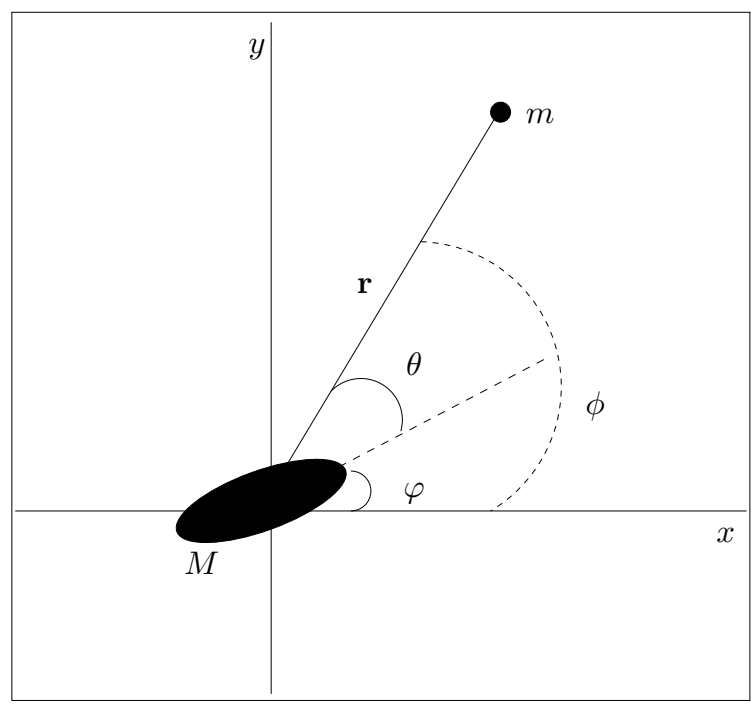

Figure 1. Gravitational interaction of a rigid body and a sphere in the plane.

the system is described by the Lagrangian functional

$$
L(\mathbf{r}, \varphi, \dot{\mathbf{r}}, \dot{\varphi})=\frac{1}{2}\|\dot{\mathbf{r}}\|^{2}+\frac{1}{2} I_{z z} \dot{\varphi}^{2}-V(\mathbf{r}, \varphi)
$$

where $\|\cdot\|$ denotes the Euclidean norm and $V(\cdot)$ is the gravitational potential of the system. The Legendre transform, $\left(\mathbf{p}, p_{\varphi}\right)=\left(\dot{\mathbf{r}}, I_{z z} \dot{\varphi}\right)$, gives the Hamiltonian formulation of the problem:

$$
H=\frac{1}{2}\|\mathbf{p}\|^{2}+\frac{1}{2 I_{z z}} p_{\varphi}^{2}+V(\mathbf{r}, \varphi) .
$$

We assume that the axes of the ellipsoid, $(\alpha, \beta, \gamma)$, are ordered as $0<\gamma \leq \beta \leq \alpha=1$ and that the " $\gamma$ axis" is orthogonal to the plane of motion. Thus, the longest axis of the ellipsoid is taken as the unit of length. The unit of time is taken such that $G m M=1$. The mass parameter of the system will be denoted $\nu=m /(m+M)$, and then the moment of inertia of the ellipsoid is $I_{z z}=\left(1+\beta^{2}\right) /(5 \nu)$. To write the mutual gravitational potential function of the ellipsoid and sphere, we use Ivory's theorem [25, 38]:

$$
V(\mathbf{r}, \varphi)=V(r, \theta)=-\frac{3}{4} \int_{\lambda(r, \theta)}^{+\infty} \Phi(r, \theta ; u) \frac{d u}{\Delta(u)},
$$

where $r=\|\mathbf{r}\|, \theta=\phi-\varphi$,

$$
\Phi(r, \theta ; u)=1-\frac{r^{2} \cos ^{2} \theta}{1+u}-\frac{r^{2} \sin ^{2} \theta}{\beta^{2}+u}
$$

$\Delta(u)=\sqrt{(1+u)\left(\beta^{2}+u\right)\left(\gamma^{2}+u\right)}$, and $\lambda(r, \theta)>0$ is the largest root of $\Phi(r, \theta ; \lambda(r, \theta))=0$. 
This system still has an overall rotational symmetry; that is, it is invariant under rotations in the plane of motion. In other words, the action of the symmetry group $S O(2)$ leaves the system invariant. Hence, to reduce the dimensionality of the problem, we apply the abelian reduction reviewed in the appendix.

For this purpose, by introducing polar coordinates and relative angles (see [12]), we write the Hamiltonian function in a much more convenient way:

$$
H=\frac{1}{2} p_{r}^{2}+\left(\frac{1}{2 r^{2}}+\frac{1}{2 I_{z z}}\right) p_{\theta}^{2}+\frac{1}{2 I_{z z}} p_{\varphi}^{2}-\frac{1}{I_{z z}} p_{\theta} p_{\varphi}+V(r, \theta)
$$

where $p_{\theta}=r^{2} \dot{\theta}+r^{2} \dot{\varphi}$ and $p_{\varphi}=r^{2} \dot{\theta}+\left(r^{2}+I_{z z}\right) \dot{\varphi}$. Notice that $\varphi$ is a cyclic variable of the Hamiltonian (2.4), and therefore its conjugate momentum $p_{\varphi}$ is conserved (Noether's theorem).

We then apply cotangent bundle reduction (see section A.2): The momentum map is given by $\mathbf{J}\left(r, \theta, \varphi, p_{r}, p_{\theta}, p_{\varphi}\right)=p_{\varphi}$ and corresponds to the total angular momentum of the system in the new coordinates. The locked inertia tensor (instantaneous inertia tensor when the relative motion of the two-body system is locked) is $\mathbb{I}(r, \theta, \varphi)=r^{2}+I_{z z}$. The mechanical connection is the 1 -form given by $\mathcal{A}(r, \theta, \varphi)=\frac{r^{2}}{r^{2}+I_{z z}} d \theta+d \varphi$. For a fixed angular momentum $p_{\varphi}=\mu$, we finally perform the shift from $\mathbf{J}^{-1}(\mu)$ to $\mathbf{J}^{-1}(0)$ as

$$
\tilde{p}_{r}=p_{r}, \quad \tilde{p}_{\theta}=p_{\theta}-\frac{\mu r^{2}}{r^{2}+I_{z z}}, \quad \tilde{p}_{\varphi}=0 .
$$

The reduced Hamiltonian in $\mathbf{J}^{-1}(0) / S^{1}$ has only two degrees of freedom,

$$
H_{\mu}(r, \theta)=\frac{1}{2} \tilde{p}_{r}^{2}+\frac{1}{2}\left(\frac{1}{r^{2}}+\frac{1}{I_{z z}}\right) \tilde{p}_{\theta}^{2}+V_{\mu}(r, \theta)
$$

and $V_{\mu}(r, \theta)$ is the amended potential,

$$
V_{\mu}(r, \theta)=\frac{\mu^{2}}{2\left(r^{2}+I_{z z}\right)}-\frac{3}{4} \int_{\lambda(r, \theta)}^{+\infty} \Phi(r, \theta ; u) \frac{d u}{\Delta(u)},
$$

where $\Phi(r, \theta ; u), \Delta(u)$, and $\lambda(r, \theta)>0$ are as defined before, and $\mu \in \mathbb{R}$ is the total angular momentum (fixed). The reduced symplectic form is noncanonical and given by

$$
\omega_{\mu}=d r \wedge d \tilde{p}_{r}+d \theta \wedge d \tilde{p}_{\theta}-\frac{2 \mu I_{z z} r}{\left(r^{2}+I_{z z}\right)^{2}} d r \wedge d \theta .
$$

The equations of motion in the reduced space can be easily derived from $\mathbf{i}_{\left(\dot{x}^{k} \partial_{x^{k}}+\dot{y}_{k} \partial_{y_{k}}\right)} \omega_{\mu}=$ $d H_{\mu}$, where $(x, y)$ denote the configuration-momenta conjugate pair and $\mathbf{i}_{X} \Omega$ is the interior product (or contraction) of the vector field $X$ and the 1 -form $\Omega$ :

$$
\begin{array}{ll}
\dot{r}=\tilde{p}_{r}, & \dot{\tilde{p}}_{r}=\frac{\tilde{p}_{\theta}^{2}}{r^{3}}-\frac{\partial V_{\mu}(r, \theta)}{\partial r}+\frac{2 \mu I_{z z} r}{\left(r^{2}+I_{z z}\right)^{2}}\left(\frac{1}{r^{2}}+\frac{1}{I_{z z}}\right) \tilde{p}_{\theta}, \\
\dot{\theta}=\left(\frac{1}{r^{2}}+\frac{1}{I_{z z}}\right) \tilde{p}_{\theta}, & \dot{\tilde{p}}_{\theta}=-\frac{\partial V_{\mu}(r, \theta)}{\partial \theta}-\frac{2 \mu I_{z z} r}{\left(r^{2}+I_{z z}\right)^{2}} \tilde{p}_{r} .
\end{array}
$$


2.2. Relative equilibria. We are now interested in finding the stable (we will make precise later on in which sense) fixed points of the reduced equations. The relative equilibria (or fixed points of the reduced equations) satisfy [27]:

$$
\tilde{p}_{r}=\tilde{p}_{\theta}=\frac{\partial V_{\mu}}{\partial r}=\frac{\partial V_{\mu}}{\partial \theta}=0 .
$$

The first two equations give

$$
\begin{aligned}
& p_{r}=0, \\
& p_{\theta}=\frac{\mu r^{2}}{r^{2}+I_{z z}},
\end{aligned}
$$

and the last two give

$$
\begin{aligned}
\frac{3}{2} r\left(\cos ^{2} \theta R_{\alpha}+\right. & \left.\sin ^{2} \theta R_{\beta}\right)-\frac{\mu^{2} r}{\left(r^{2}+I_{z z}\right)^{2}}=0, \\
-\frac{3}{4} r^{2} \sin 2 \theta\left(R_{\alpha}-R_{\beta}\right) & =0,
\end{aligned}
$$

where $R_{\alpha}$ and $R_{\beta}$ denote the elliptic integrals

$$
R_{\alpha}=\int_{\lambda(r, \theta)}^{+\infty} \frac{d u}{(1+u) \Delta(u)}, \quad R_{\beta}=\int_{\lambda(r, \theta)}^{+\infty} \frac{d u}{\left(\beta^{2}+u\right) \Delta(u)} .
$$

2.2.1. Spheroid. First, let us look at the case where the in-plane axes of the ellipsoid are equal. In this case, $0<\gamma<\beta=\alpha=1$, and thus $R_{\alpha}=R_{\beta}$. Then, (2.9) is satisfied automatically $\forall \theta \in \mathbb{T}$.

From (2.8) and assuming $r>1$,

$$
\frac{\mu^{2}}{\left(r^{2}+I_{z z}\right)^{2}}=\frac{3}{2} R_{\alpha}
$$

where in this case

$$
R_{\alpha}=\int_{r^{2}-1}^{+\infty} \frac{d u}{(1+u)^{2} \sqrt{\gamma^{2}+u}}
$$

In relative equilibria, the distance between the two bodies is constant ( $r \equiv$ constant). We can thus define the following parameter:

$$
\omega \equiv \frac{\mu}{r^{2}+I_{z z}}
$$

The relative equilibria can be seen in the unreduced space as uniformly rotating systems. Then, $\omega$ is precisely the angular velocity of this rotating system.

In this degenerate case, the elliptic integral $R_{\alpha}$ is trivially integrable, and we obtain the following condition for relative equilibria: Given $r>1$ and $\gamma<1$,

$$
\omega^{2}=3 \pi-\frac{3 \sqrt{r^{2}-1+\gamma^{2}}}{4\left(1-\gamma^{2}\right) r^{2}}-\frac{3}{2\left(1-\gamma^{2}\right)^{3 / 2}} \arctan \sqrt{\frac{r^{2}-1+\gamma^{2}}{1-\gamma^{2}}} .
$$




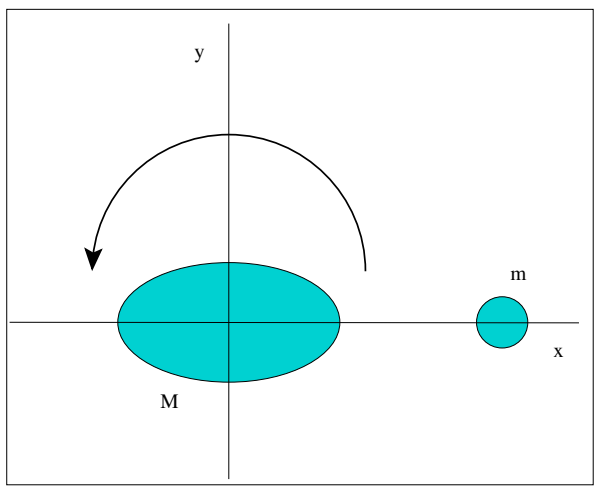

(a)

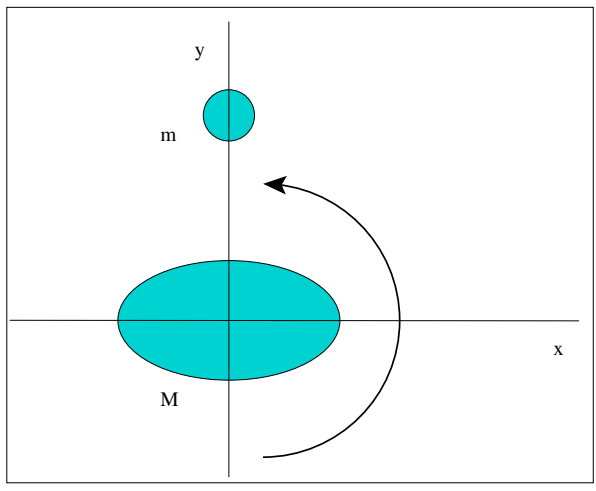

(b)

Figure 2. Two types of relative equilibria for the planar ellipsoid-sphere Full 2-Body Problem. (a) LongAxis Equilibrium (LAE). (b) Short-Axis Equilibrium (SAE).

Note: For fixed $\gamma<1$ and $r>1$, we have a degenerate "circle" of relative equilibria $(\forall \theta \in \mathbb{T}$ ) with the binary rotating (in an inertial frame) at a constant angular velocity $\omega$ satisfying (2.11). Also, for fixed mass parameter $\nu$, we can compute $I_{z z}=2 /(5 \nu)$ and then the angular momentum $\mu$ from (2.10).

2.2.2. Triaxial ellipsoid. Now, we focus on the triaxial ellipsoid, where $0<\gamma \leq \beta<\alpha=$ 1. Here, $R_{\alpha} \neq R_{\beta}$, and (2.9) yields $\sin 2 \theta=0$, which means that relative equilibria satisfy

$$
\theta=k \frac{\pi}{2} \quad \text { for any } k \in \mathbb{Z} \text {. }
$$

Geometrically, we can distinguish between two types of solutions [40]:

1. LAE (long-axis equilibria) when $\theta=0$ or $\pi$ (see Figure 2(a)), and

2. SAE (short-axis equilibria) when $\theta= \pm \frac{\pi}{2}$ (see Figure $2(\mathrm{~b})$ ).

Again, defining the angular velocity of the system as in (2.10), we obtain the following relations between $\omega$ and $r$ :

1. LAE:

$$
\omega^{2}=\frac{3}{2} \int_{r^{2}-1}^{+\infty} \frac{d u}{(1+u) \Delta(u)}
$$

2. SAE:

$$
\omega^{2}=\frac{3}{2} \int_{r^{2}-\beta^{2}}^{+\infty} \frac{d u}{\left(\beta^{2}+u\right) \Delta(u)}
$$

where recall that $\Delta(u)=\sqrt{(1+u)\left(\beta^{2}+u\right)\left(\gamma^{2}+u\right)}$. Here, for fixed $0<\gamma \leq \beta<1$ and $R \geq 1$, there is a fixed point with coordinates $(r, \theta)=(R, k \pi / 2)$ for an angular velocity $\omega$ satisfying (2.12) for $k \bmod 2=0$, or $(2.13)$ for $k \bmod 2=1$. Moreover, given the mass parameter, $\nu$, one can compute the ellipsoid's moment of inertia $I_{z z}=\left(1+\beta^{2}\right) /(5 \nu)$ and, using $(2.10)$, the angular momentum of the system $\mu$. 
2.3. Stability of relative equilibria: Energy-momentum method. We apply the energymomentum method of Simo, Lewis, and Marsden [44] to study the stability of the relative equilibria that we have just found. According to this method (see also [31, 3]), to carry out the stability analysis of the LAE and SAE, we must compute $\delta^{2} V_{\mu}$ on the subspace orthogonal to the group $G_{\mu}$-orbit (in this case, $G_{\mu}=S O(2)=S^{1}$ ).

From (2.6), it is easy to see that

$$
\delta^{2} V_{\mu}=\left(\begin{array}{cc}
A & 0 \\
0 & B
\end{array}\right)
$$

for $\theta=k \frac{\pi}{2}$ (LAE and SAE) and where $A, B \in \mathbb{R}$ and their values depend, of course, on the particular relative equilibrium.

In the spheroid case, $\alpha=\beta$, and thus

$$
A=\frac{4 r^{2} \omega^{2}}{r^{2}+I_{z z}}-\frac{3}{r^{2} \sqrt{r^{2}+\gamma^{2}-1}},
$$

where $\omega$ satisfies $(2.11)$ and $B=0$. This is due to the fact that the relative equilibria are degenerate. Therefore, the energy-momentum method is not conclusive for the "circle" of relative equilibria in the spheroid-sphere particular case.

1. Stability of LAEs. In this case, $\theta=0$ or $\pi$ and thus

$$
\begin{aligned}
& B=-\frac{3}{2} r^{2}\left(R_{\alpha}-R_{\beta}\right), \\
& A=\frac{4 r^{2} \omega^{2}}{r^{2}+I_{z z}}-\frac{3}{r \sqrt{\left(r^{2}+\beta^{2}-1\right)\left(r^{2}+\gamma^{2}-1\right)}},
\end{aligned}
$$

where $\omega$ is given by (2.12). As $\alpha>\beta, R_{\alpha}<R_{\beta}$ and thus $B>0$. Then,

(a) if $A>0$, the LAE is (linearly and nonlinearly) stable;

(b) if $A<0$, the LAE is unstable (the index is odd [31, 35]).

In Figure 3, we show some ranges for the parameter values that give stability of the LAE.

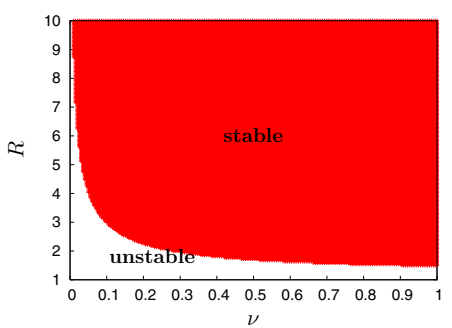

(a)

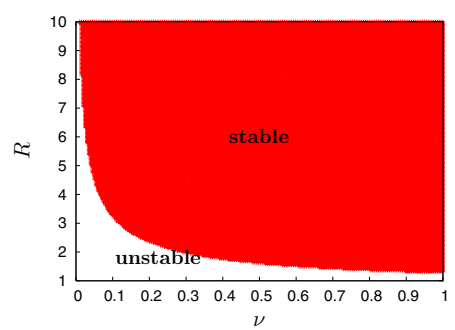

(b)

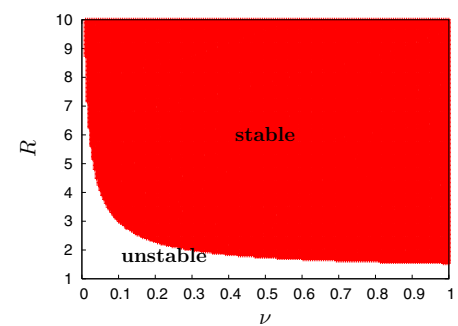

(c)

Figure 3. LAE. Nonlinear stability (red zone) and instability (white zone) of the long-axis relative equilibria of the binary with respect to the mass parameter $\nu$ and the distance between the two bodies $R$ for some values of the ellipsoid axes: (a) $\beta=\gamma=0.5$, (b) $\beta=\gamma=0.8$, (c) $\beta=0.5$ and $\gamma=0.25$. 
2. Stability of SAEs. In this case, $\theta= \pm \frac{\pi}{2}$ and

$$
\begin{aligned}
& B=\frac{3}{2} r^{2}\left(R_{\alpha}-R_{\beta}\right), \\
& A=\frac{4 r^{2} \omega^{2}}{r^{2}+I_{z z}}-\frac{3}{r \sqrt{\left(r^{2}+1-\beta^{2}\right)\left(r^{2}+\gamma^{2}-\beta^{2}\right)}},
\end{aligned}
$$

where $\omega$ is given by (2.13). As $\alpha>\beta, R_{\alpha}<R_{\beta}$ and thus $B<0$. Then,

(a) if $A>0$, the SAE is unstable (the index is odd [31, 35]);

(b) if $A<0$, the stability of the SAE requires further analysis. In this case, we study their spectral stability by computing numerically the eigenvalues of the linearized vector field at the fixed points of the reduced system.

In Figure 4, we show ranges for the parameter values for which the SAE are spectrally stable, unstable, or complex unstable.

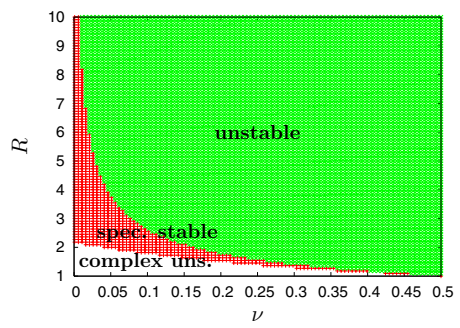

(a)

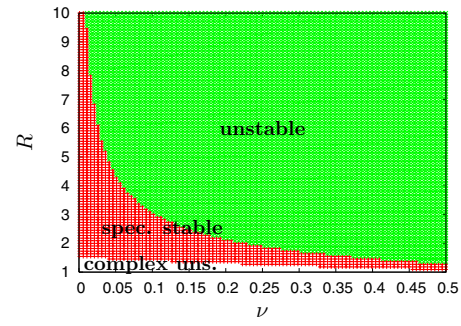

(b)

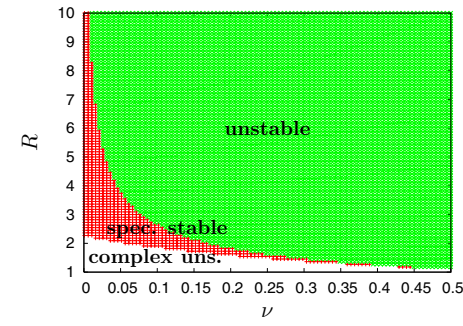

(c)

Figure 4. SAE. Unstable zone (green), complex unstable zone (white), and spectrally stable zone (red) of the short-axis relative equilibria of the binary with respect to the mass parameter $\nu$ and the distance between the two bodies $R$ for some values of the ellipsoid axes: (a) $\beta=\gamma=0.5$, (b) $\beta=\gamma=0.8$, (c) $\beta=0.5$ and $\gamma=0.25$.

3. RF3BP. We focus now on a satellite influenced by the gravitational potential of the binary. To model the motion of this satellite, we assume that the binary is in a relative equilibrium, and we use the study performed in the last section. As the relative equilibria found in section 2.2 are periodic orbits for the unreduced system, we will write the equations of motion for the satellite in a rotating reference in which the asteroid pair is fixed.

We consider binaries such that the rigid body (ellipsoid) is "big" and the spherical body is "small." Hence, we assume that $\nu \ll 1$. This situation is thought to be the most common in the main belt [33]. Moreover, as for $\nu \ll 1$ and moderate $R$ values the SAE are spectrally stable, we will also assume that the binary is moving in this particular solution of the F2BP. For a study of the motion of a spacecraft near a binary in LAE, see [41].

We will now derive the equations of motion for a satellite orbiting a binary in SAE. We start by assuming that the barycenter of the system is at the origin. As in an inertial reference frame, the SAE is a uniformly rotating motion [12], and we write the equations for the spacecraft in a rotating frame for which the two massive bodies, ellipsoid and sphere, are fixed. More concretely, we choose a reference system where the centers of mass of the ellipsoid and sphere are at the configuration points $(-\nu R, 0,0)$ and $((1-\nu) R, 0,0)$, respectively; see Figure 5. In this case, note that the longest axis of the ellipsoid is parallel to the $y$ axis. 


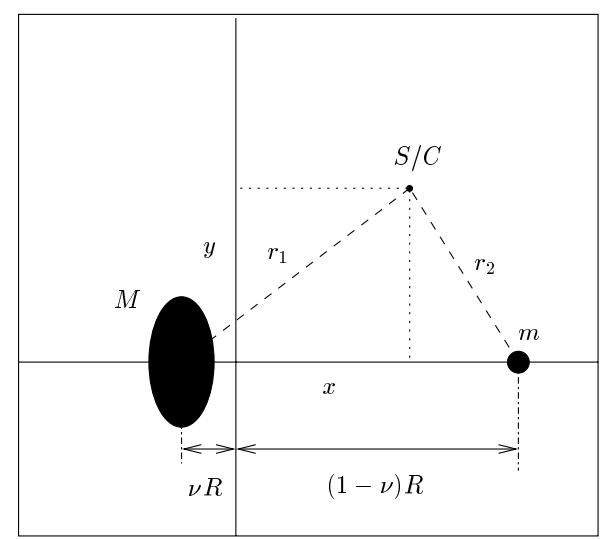

Figure 5. Model for the spacecraft motion around the binary asteroid. The asteroid pair is assumed to be in $S A E$, and the equations of motion for the spacecraft are written in a rotating frame.

Even though the uniformly rotating motion of the binary is in the $x y$-plane, we will consider that the satellite is allowed to move in the entire three-dimensional $x y z$-configuration space. The equations of motion for the spacecraft in this situation can be constructed similarly to the RTBP equations [45]. They allow a Hamiltonian formulation and can be obtained from the following Hamiltonian function:

$$
\begin{aligned}
H\left(p_{x}, p_{y}, p_{z}, x, y, z\right) & =\frac{1}{2}\left(p_{x}^{2}+p_{y}^{2}+p_{z}^{2}\right)+\omega\left(y p_{x}-x p_{y}\right)+V(x, y, z), \\
V(x, y, z) & =-\frac{3(1-\nu)}{4} \int_{\lambda(x, y, z)}^{+\infty} \Phi(x, y, z ; u) \frac{d u}{\Delta(u)}-\frac{\nu}{r_{2}},
\end{aligned}
$$

where $(x, y, z)$ is the position of the spacecraft in the rotating reference frame, $\left(p_{x}, p_{y}, p_{z}\right)$ are the conjugate momenta, and $\omega$ is the angular velocity of the rotating system (2.13). We define $R$ to be the distance between the ellipsoid and sphere barycenters. Then, $r_{2}^{2}=$ $(x-(1-\nu) R)^{2}+y^{2}+z^{2}$. The gravitational potential coming from the ellipsoid is computed as before but considering that now the reference frame is tilted by $90^{\circ}$. Then, we compute $V(x, y, z)$ using

$$
\Phi(x, y, z ; u)=1-\frac{(x+\nu R)^{2}}{\beta^{2}+u}-\frac{y^{2}}{1+u}-\frac{z^{2}}{\gamma^{2}+u}
$$

and $\Delta(u)=\sqrt{(1+u)\left(\beta^{2}+u\right)\left(\gamma^{2}+u\right)}$. Finally, $\lambda(x, y, z)>0$ is defined implicitly as the largest positive root of $\Phi(x, y, z ; \lambda(x, y, z))=0$.

In this case, the symplectic form of the system is canonical, and the equations of motion for the spacecraft are very easy to derive. From now on, we will denote this problem the 
RF3BP, and the differential equations are

$$
\begin{aligned}
& \dot{x}=p_{x}+\omega y, \quad \dot{p_{x}}=\omega p_{y}-(1-\nu)(x+\nu R) R_{\beta}-\frac{\nu(x-(1-\nu) R)}{r_{2}^{3}}, \\
& \dot{y}=p_{y}-\omega x, \quad \dot{p_{y}}=-\omega p_{x}-(1-\nu) y R_{\alpha}-\frac{\nu y}{r_{2}^{3}}, \\
& \dot{z}=p_{z}, \\
& \dot{p}_{z}=-(1-\nu) z R_{\gamma}-\frac{\nu z}{r_{2}^{3}}
\end{aligned}
$$

where

$$
\begin{aligned}
R_{\alpha} & =\frac{3}{2} \int_{\lambda(x, y, z)}^{+\infty} \frac{d u}{(1+u) \Delta(u)}, \\
R_{\beta} & =\frac{3}{2} \int_{\lambda(x, y, z)}^{+\infty} \frac{d u}{\left(\beta^{2}+u\right) \Delta(u)}, \\
R_{\gamma} & =\frac{3}{2} \int_{\lambda(x, y, z)}^{+\infty} \frac{d u}{\left(\gamma^{2}+u\right) \Delta(u)} .
\end{aligned}
$$

To compute these elliptic integrals, we use Carlson's elliptic integral of the second kind [4]:

$$
\begin{aligned}
& R_{\alpha}(x, y, z)=R_{D}\left(\beta^{2}+\lambda(x, y, z), \gamma^{2}+\lambda(x, y, z), 1+\lambda(x, y, z)\right), \\
& R_{\beta}(x, y, z)=R_{D}\left(1+\lambda(x, y, z), \gamma^{2}+\lambda(x, y, z), \beta^{2}+\lambda(x, y, z)\right), \\
& R_{\gamma}(x, y, z)=R_{D}\left(1+\lambda(x, y, z), \beta^{2}+\lambda(x, y, z), \gamma^{2}+\lambda(x, y, z)\right),
\end{aligned}
$$

where $R_{D}(x, y, z)=\frac{3}{2} \int_{0}^{+\infty}(t+x)^{-1 / 2}(t+y)^{-1 / 2}(t+z)^{-3 / 2} d t$.

3.1. Substitutes of the Lagrangian points. As was mentioned before, it is well known [45] that the RTBP has five equilibrium points. Three of them lie on the $x$ axis and are called collinear or Eulerian points, and two of them, the triangular or Lagrangian points (also known as $L_{4}$ and $L_{5}$ ), form an equilateral triangle with the primaries. Here, we are interested in investigating how the rigid-body (ellipsoid) affects the position and stability of these triangular points. See Figure 6.

The fixed points of the RF3BP satisfy the following set of equations:

$$
\begin{aligned}
& p_{x}=-\omega y, \quad \omega^{2} x=(1-\nu)(x+\nu R) R_{\beta}+\frac{\nu(x-(1-\nu) R)}{r_{2}^{3}}, \\
& p_{y}=\omega x, \quad \omega^{2} y=(1-\nu) y R_{\alpha}+\frac{\nu y}{r_{2}^{3}}, \\
& p_{z}=0, \\
& 0=(1-\nu) z R_{\gamma}+\frac{\nu z}{r_{2}^{3}} \text {. }
\end{aligned}
$$

There are three solutions satisfying $y=z=0$ that correspond to the "perturbed" collinear points. These equilibria are unstable [2] and therefore not suitable for constructing orbits in which to park a spacecraft. A priori, one could think that the collinear points of the RF3BP can have applications similar to the RTBP [29], such as the Genesis Discovery Mission, and 


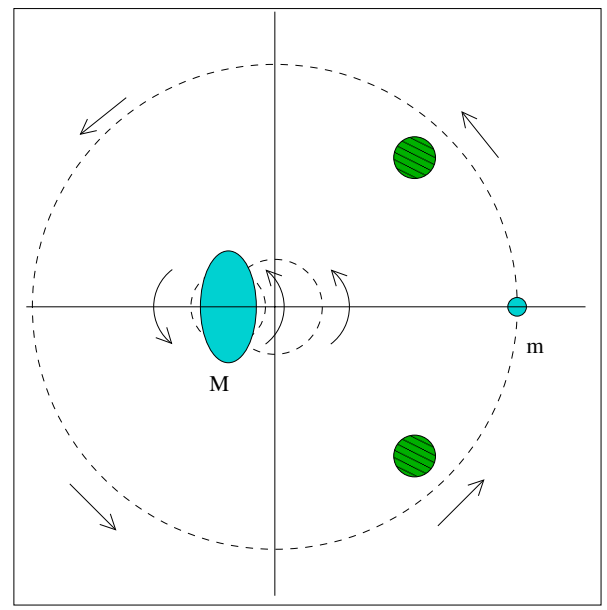

Figure 6. Schematic picture of the stability zones near the Lagrangian points of the RF3BP.

that it can be cheap to park a spacecraft near them by using the so-called center manifold $[18,15]$. But this is not true in the current study. The difference is that in the binary asteroid problem the time scale is much shorter. This makes things such that the correction maneuvers, necessary to compensate the normal instability of the orbits, have to be performed too often to be feasible in practice.

We look for solutions such that $z=0$ and $y \neq 0$. If there were no rigid-body effects, these fixed points would correspond to the RTBP $L_{4}$ and $L_{5}$. They are thus the substitutes of the Lagrangian points and satisfy

$$
\begin{aligned}
\omega^{2} x & =(1-\nu)(x+\nu R) R_{\beta}+\frac{\nu(x-(1-\nu) R)}{r_{2}^{3}}, \\
\omega^{2} & =(1-\nu) R_{\alpha}+\frac{\nu}{r_{2}^{3}} .
\end{aligned}
$$

We have numerically solved these equations for certain values of the parameters $\beta, \gamma, \nu$, and $R$ by means of Newton's method. In Figure 7, we plot the $(x, y)$-projection of these fixed points for $y>0$ (there is a symmetric solution at $-y$ ) after shifting and rescaling in such a way that the ellipsoid is centered at $(0,0)$ and the sphere at $(1,0)$. After fixing $\beta$ and $\gamma$, we vary the mass parameter $\nu$ from $10^{-3}$ to 0.5 and the distance between primaries from $R=1$ to $R=10$. Every red dot in Figure 7 corresponds to the substitute of the $L_{4}$ fixed point for a particular set of parameter values. More concretely, the red point at the bottom corner of every figure corresponds to $\nu=10^{-3}$ and $R=1$; the point at the top-left corner to $\nu=0.5$ and $R=1$; and, the point at the top-right corner to $\nu=0.5$ and $R=10$. See caption for more details.

Recall that, in these units, the Lagrangian point $\left(L_{4}\right)$ is at the position $(x, y)=(0.5, \sqrt{3} / 2)$. From the pictures, we can see that for small $\nu$ (big rigid-body) and short $R$ (the two bodies are close) the position of the fixed point deviates a lot from the $L_{4}$ position. For larger $\nu$ values and moderate distances between the primaries, the fixed point is closer to the RTBP triangular region. 


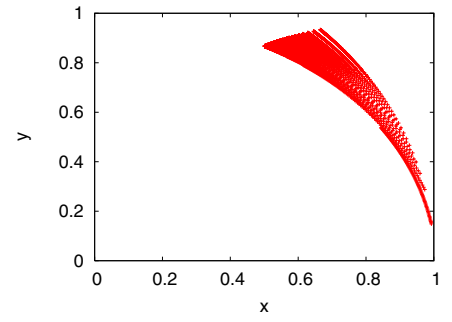

(a)

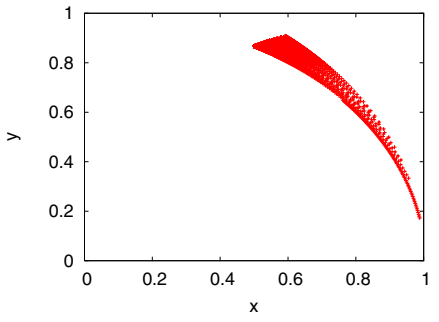

(b)

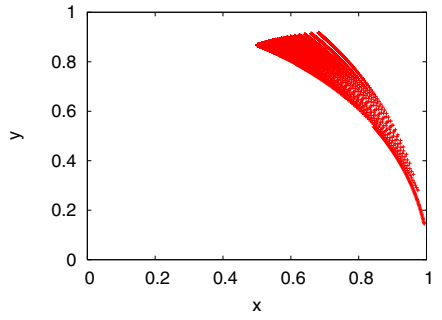

(c)

Figure 7. Actual positions $(x, y, 0)$ of the substitutes of the Lagrangian point $L_{4}$ in the xy-plane when the mass parameter is varied between $10^{-3}$ and 0.5 (right to left) and the distance $R$ increases from 1 to 10 (bottom to top). The positions have been shifted and rescaled in such a way that the ellipsoid is centered at $(0,0,0)$ and the sphere at $(1,0,0)$. (a) $\beta=\gamma=0.5$, (b) $\beta=\gamma=0.8$, (c) $\beta=0.5$ and $\gamma=0.25$.

3.2. Spectral stability of the equilibrium points. We focus now on the study of the stability of the substitutes of the Lagrangian points in the RF3BP. In this section, we consider their spectral stability, i.e., stability w.r.t. eigenvalues of the linearized vector field. In section 4, we will study the nonlinear stability by means of a numerical method.

We begin with the linearization of the system (3.1) at the equilibrium point that substitutes $L_{4}$. We then compute numerically its eigenvalues. Similarly at the RTBP, this study shows that for "small" mass parameter values $(\nu \ll 1)$, the fixed points are spectrally stable and, for larger $\nu$, they are complex unstable, although in certain cases the critical value is larger than the Routh mass [41].

In Figure 8, we show some examples of the spectral stability behavior of the perturbed triangular fixed points of the RF3BP for different types of ellipsoids. We also superimpose on these pictures the stability zone of the SAE of the corresponding binary system.

In the next section, to perform a numerical global study of the stability region around the perturbed Lagrangian points in the RF3BP, we will be interested in the cases for which the underlying binary system is in a stable SAE and for which the corresponding triangular fixed point is spectrally stable. Thus, we will look at the intersection of the green and red zones in Figure 8.

4. Global dynamics around the Lagrangian points. In this section, the refined Fourier analysis method (see [16]) is used to study the dynamics around the tadpole region of the Lagrangian points. First, we obtain a global dynamical picture of the neighborhood of the fixed points. Later we identify trajectories that are very close to quasi-periodic motion and place the spacecraft in them, to simulate its dynamics while it is in a position in which to make observations of the binary system.

As the computations involved in this section are very intensive, we do not intend to perform a systematic study of the dynamics in terms of the parameters (which we believe was approached in the last sections), but we will choose a particular set of parameter values close to an actual asteroid pair. We thus start by fixing the four parameters of the RF3BP in the following manner: $\beta=0.7576, \gamma=0.6314, \nu=1.16 \times 10^{-3}$, and $R=5.873$. These particular values approximate the ones of the binary Kalliope-Linus system, placed in the main-belt (see [17]). Even though observations suggest that this asteroid pair is not in SAE, 


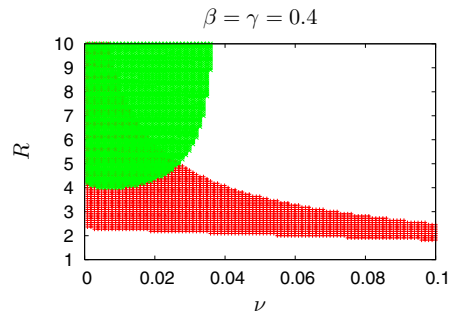

(a)

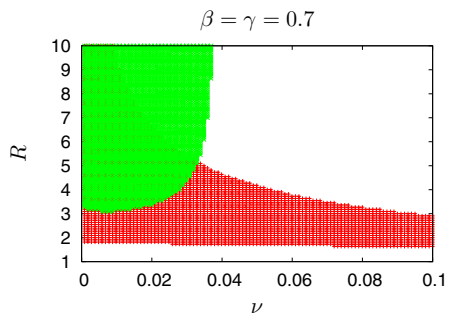

(d)

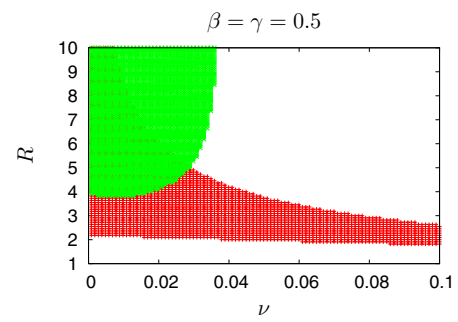

(b)

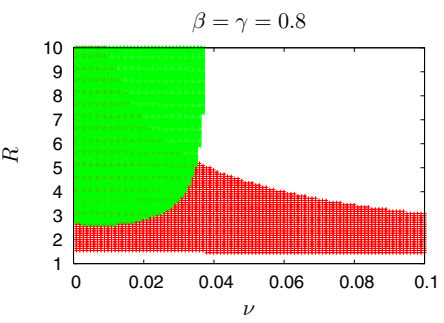

(e)

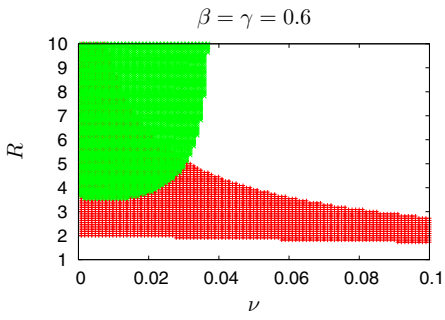

(c)

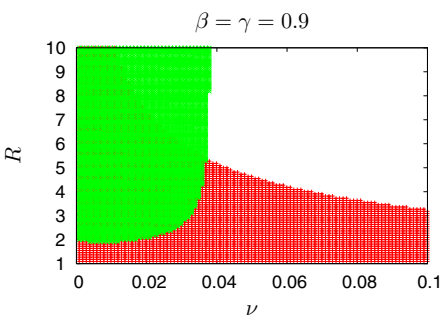

(f)

Figure 8. Green: spectrally stable zones for the perturbed triangular points in the RF3BP. Red: spectrally stable zones for the SAE of the corresponding asteroid pair. The eigenvalues are computed for fixed $\beta$ and $\gamma$, for a mass parameter $\nu \in[0,0.1]$, and for a distance between the primaries $R \in[1,10]$. The fixed values of the ellipsoid's axes are (a) $\beta=\gamma=0.4$, (b) $\beta=\gamma=0.5$, (c) $\beta=\gamma=0.6$, (e) $\beta=\gamma=0.7$, (f) $\beta=\gamma=0.8$, (g) $\beta=\gamma=0.9$.

as an illustration of how the method works we will construct the model for the spacecraft assuming that the underlying binary with these parameter values is actually in SAE. Here, we use the construction of the RF3BP as in section 3. In this case, the uniform rotation of the binary in SAE is $\omega=7.01844077933 \times 10^{-2}$, which corresponds to a complete revolution every 3.6 terrestrial days.

For this particular set of parameter values, the triangular points of the RF3BP are elliptic fixed points. From the eigenvalues of the linearized system, it is possible to obtain the frequencies of the normal oscillators at the equilibrium point:

$$
\begin{aligned}
& \omega_{1}=1.72741550738 \times 10^{-2}, \\
& \omega_{2}=6.76474915889 \times 10^{-2}, \\
& \omega_{3}=7.05487253096 \times 10^{-2} .
\end{aligned}
$$

Under generic conditions, the Kolmogorov-Arnold-Moser (KAM) theorem [19, 1, 34] (see [23] or [43] for a survey) ensures the existence of many quasi-periodic trajectories in a small neighborhood of these fixed points. In practice, the domain of applicability of the KAM theorem is much smaller than the actual stability region, which can be extended far from the elliptic point $[32,14,8,9]$.

In this section, we explore numerically this stability region near the Lagrangian points of the RF3BP by means of the frequency analysis method. 


\subsection{Frequency analysis and global dynamics. Let}

$$
f(t)=\sum_{k \in \mathbb{Z}^{m}} a_{k} e^{i<k, \nu>t}, \quad a_{k} \in \mathbb{C},
$$

be a quasi-periodic function for which we know a table of equidistant values in the time span $[0, T]$. The frequency analysis algorithm will provide the values of the frequencies $\nu_{k}$ and the amplitudes $\tilde{a}_{k}$ of a function $\tilde{f}(t)=\sum \tilde{a}_{k} e^{i \nu_{k} t}$ that approximates $f(t)$ in $[0, T]$. The actual method used for approximating the frequencies is the one given by [16]. The procedure consists, basically, of equating the discrete Fourier transforms of the sampled initial data and of the quasi-periodic approximation. For an introduction to the frequency analysis (FA) method, see [21].

With the help of the FA method, we construct pictures of the global phase space dynamics near the Lagrangian fixed points. As the phase space is six-dimensional (the RF3BP is a three degrees of freedom problem), we need to reduce dimensionality by fixing some coordinates to constants. We thus study particular slices of phase space that are relevant for the dynamics.

The practical implementation is as follows. First, we transform the Cartesian coordinates of $(3.1),\left(x, y, z, p_{x}, p_{y}, p_{z}\right)$, to spherical coordinates $\left(\rho, \theta, \lambda, p_{\rho}, p_{\theta}, p_{\lambda}\right)$ centered at the rigidbody barycenter by means of the canonical change of variables given by the generating function

$$
W\left(p_{x}, p_{y}, p_{z}, \rho, \theta, \lambda\right)=-\rho\left(p_{x} \cos \theta \cos \lambda+p_{y} \sin \theta \cos \lambda+p_{z} \sin \lambda\right) .
$$

This change is useful in the visualization of the global dynamics, since it is well known that the stability region near the RTBP Lagrangian points is of the "banana shape" [32, 13, 8, 9]. Thus, we believe that they are a good set of coordinates with which to investigate the neighborhood of the RF3BP triangular points as well.

The spherical coordinates of the upper $(y>0)$ triangular point for the Kalliope-Linus system are

$$
\begin{array}{ll}
\rho^{t}=1.0012900026, & p_{r}^{t}=0.0000000000, \\
\theta^{t}=31.207021475^{\circ}, & p_{\theta}^{t}=1.0025816692, \\
\lambda^{t}=0.0000000000, & p_{\lambda}^{t}=0.0000000000 .
\end{array}
$$

The zone of interest is a sufficiently large neighborhood of this point in phase space, and one can fix some coordinates to study some particular slices. We zoom into a window in the $(\rho, \theta)$-space enclosing this triangular point by fixing all the momenta equal to the momenta of the fixed point, $\left(p_{\rho}, p_{\theta}, p_{\lambda}\right)=\left(p_{\rho}^{t}, p_{\theta}^{t}, p_{\lambda}^{t}\right)$, and by choosing different slices for the inclination $\lambda=\Lambda$, where $\Lambda$ is a constant. In the experiments, we choose $\Lambda=0^{\circ}, 1^{\circ}, 2^{\circ}, \ldots, 8^{\circ}$.

Inside the $(\rho, \theta)$-window we pick a regular mesh of 57,600 initial conditions and integrate them in the interval of time $[0, T]$, with $T=50,000$ adimensional units (this corresponds to about 2,000 terrestrial days in the Kalliope-Linus system). Then, we take 32,768 sample points for every trajectory and use the refined Fourier analysis [16] of this sample to evaluate the three basic frequencies (recall that the RF3BP is a three degrees of freedom system) of the orbits that we call $\omega_{1}^{(1)}, \omega_{2}^{(1)}$, and $\omega_{3}^{(1)}$. Afterwards, we repeat the integration in the interval of time $[T, 2 T]$ and recompute the frequencies. In this case, we call them $\omega_{1}^{(2)}, \omega_{2}^{(2)}$, and $\omega_{3}^{(2)}$. 
Finally, we consider the values $\delta_{j}=\left|1-\omega_{j}^{(2)} / \omega_{j}^{(1)}\right|, j=1,2,3$, as an estimation of the diffusion (see [37] and [36]) related to the orbit starting at the phase space point $\left(\rho, \theta, \lambda, p_{\rho}, p_{\theta}, p_{\lambda}\right)$. We call $\delta_{j}$ the diffusion index. The values of $\delta_{j}$ give an estimation of the chaoticity of the particular orbit. That is, if the trajectory associated with an initial condition is quasi-periodic, then $\delta_{j}$ should be zero.

In Figure 9, we show several contour plots of the function $\sigma_{j}=\log \delta_{j}$ for $j=1$ (we obtain similar pictures for the $\sigma_{2}$ and $\sigma_{3}$ cases). Every picture is performed for a fixed initial inclination $\lambda=\Lambda$, from $\Lambda=0^{\circ}$ (top-left) to $\Lambda=8^{\circ}$ (bottom-right). We plot a color depending on the value of the diffusion index: blue zones $\left(\delta_{1}<10^{-10}\right)$ correspond to initial conditions whose trajectories are close to quasi-periodic; yellow-to-red zones $\left(\delta_{1}>10^{-2}\right)$ are related to strongly irregular and escaping motion.

As we already mentioned, the top-left picture in Figure 9 corresponds to zero initial inclination, i.e., $\Lambda=0^{\circ}$. In synodic coordinates, this is the $x y$ plane of motion. As this plane is invariant under the dynamics of (3.1), all orbits starting at this particular slice will remain in this plane and thus will be coplanar to the motion of the binary. We note that the stability zone for this particular plane is quite different from the well-known "banana" region of the RTBP $[32,14,8]$. In particular, for a similar mass parameter $\nu \simeq 10^{-3}$, the stability zones near the RTBP $L_{4}$ and $L_{5}$ are extended to a much larger domain in the $x y$ plane [9].

When one increases the inclination $\Lambda$ from $0^{\circ}$ to $8^{\circ}$, we see that the stability zone shrinks rapidly until it "breaks" at about $\Lambda \sim 6.5^{\circ}$ and disappears at about $\Lambda \sim 10^{\circ}$. This is also very different from the RTBP case, since it is well known (see $[14,36]$ ) that the stable zone for the Sun-Jupiter RTBP $\left(\nu \simeq 10^{-3}\right)$ grows until $\Lambda \sim 17^{\circ}$ and does not disappear until $\Lambda>45^{\circ}$ (recall the existence of the Trojan asteroids, which can be observed at inclinations larger than $40^{\circ}$; see $\left.[7,10]\right)$.

4.2. Gallery of quasi-periodic spacecraft trajectories. We now use the global dynamical pictures obtained in the last section to effectively compute (close-to) quasi-periodic orbits near the upper triangular point of the RF3BP. These orbits will lie on near-invariant tori of dimensions 2 and 3 .

To construct these tori, we proceed as follows. First, we choose initial conditions from the global dynamical pictures that have a small associated index (we will make precise later how small). A trajectory corresponding to one of these initial conditions will remain very close to an invariant torus with frequencies $\omega_{1,2,3}$ (computed as in section 4.1). Thus, we can numerically integrate these initial conditions for a "long-enough" time and generate the (almost-) invariant torus (it would be invariant if the diffusion index was exactly zero).

In Figures 10 through 15, we show some examples of these (almost)-invariant tori. All of the images have been generated by looking for initial conditions in the global pictures of Figure 9 with a diffusion index $\delta_{1}$ smaller than $10^{-12}$. In particular, we have integrated them for a time span of $T=450,000$ adimensional units, that is, about 50 terrestrial years. We could thus send a spacecraft to one of these tori, and the satellite would orbit in this (almost) quasi-periodic orbit, without any need of extra propulsion, for at least 50 years; this is a period more than enough for a mission of this type.

More concretely, Figure 10 shows the $x y$-projections of nine planar tori. These tori lie on the zero inclination $\left(I=0^{\circ}\right)$ plane and, as this is an invariant plane for the RF3BP, spacecraft 

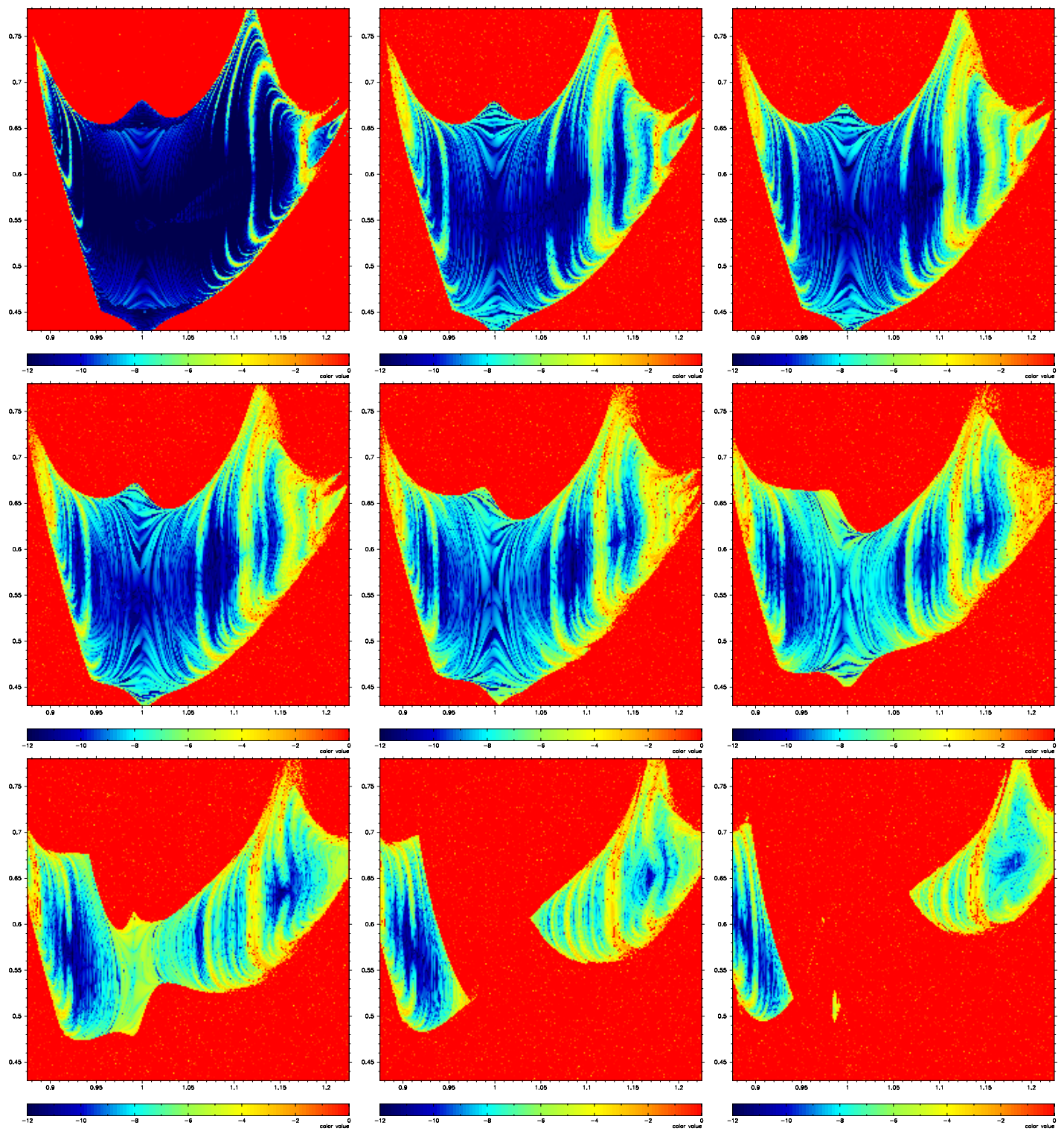

Figure 9. Global dynamics around the upper triangular point of the Kalliope-Linus system. The axes correspond to the $(\rho, \theta)$ coordinates, where the angle is in radians. The pictures show different slices of phase space for different inclinations $\Lambda$. From left to right and top to bottom, the portraits correspond to $\Lambda=0^{\circ}$, $\Lambda=1^{\circ}, \ldots, 8^{\circ}$. Blue zones are related to motion close to quasi-periodic, and hence any trajectory starting on them is likely to remain long enough in the neighborhood of the fixed point. Yellow to red zones correspond to initial conditions that escape, go to collision, or have a chaotic behavior in the time-window considered. See text for more details.

trajectories on these tori will be coplanar with the orbital motion of the binary. In Figures 11 and 12 , we plot the $x y-, x z-$, and $z \dot{z}$-projections of six three-dimensional tori with initial inclination $I=2^{\circ}$. In Figure 13, some examples of tori with initial inclination $I=5^{\circ}$ are shown. Three examples with initial inclination $I=6^{\circ}, I=7^{\circ}$, and $I=8^{\circ}$ are displayed in 

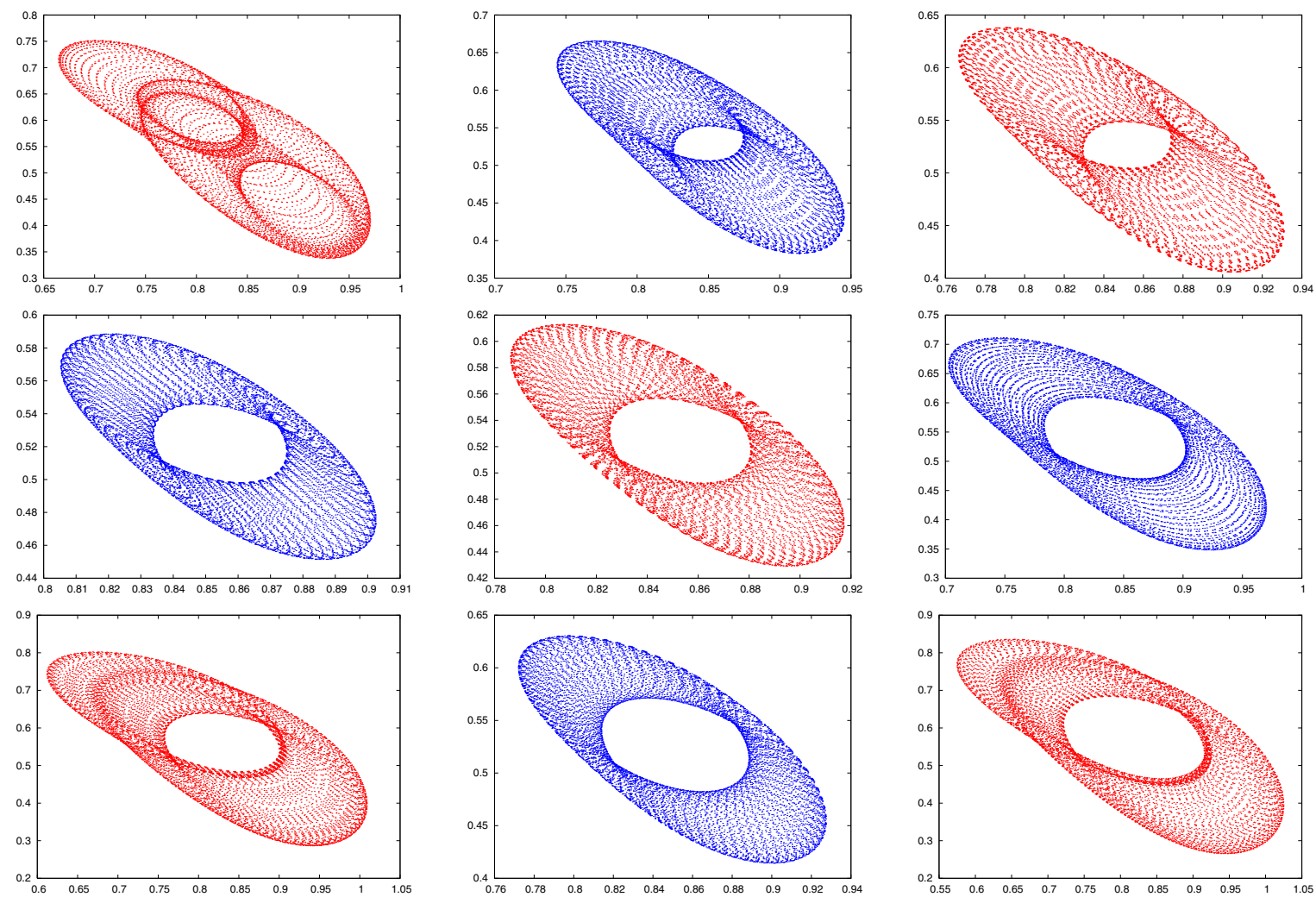

Figure 10. $x y$-projections of tori in the plane $I=0^{\circ}$.

Figure 14. Any particular orbit chosen on these tori with nonzero initial inclination can be useful for a spacecraft devoted to performing observations of the asteroid pair from different perspectives. Finally, in Figure 15, we plot the three-dimensional $x y z$-projections of some tori that appear in Figures 13 and 14. As we mentioned before, for larger inclinations the stability region vanishes, and therefore no quasi-periodic orbits are found near the Lagrangian points for $I \geq 10^{\circ}$.

5. Conclusions. In this paper, we have developed and studied a model for a satellite orbiting an asteroid pair. First, geometric mechanics was used in the modelization process of the F2BP and in studying the stability of its relative equilibria. Then, an RF3BP was constructed to describe the motion of the satellite, and numerical methods were used to study some of its global stability properties. The main tool for the numerical investigation was the frequency map analysis, which provides a very nice global view of the dynamics of this model. Moreover, this global dynamics can be used in practice to preselect initial conditions for satellite trajectories. Due to the stability properties of these particular orbits, they are very suitable for parking the spacecraft on them in such a way that there is no need to spend extra energy in the station-keeping.

Another interesting result of this paper comes from the analysis of the stability region near the Lagrangian points of the RF3BP and its comparison with the RTBP. We have seen 

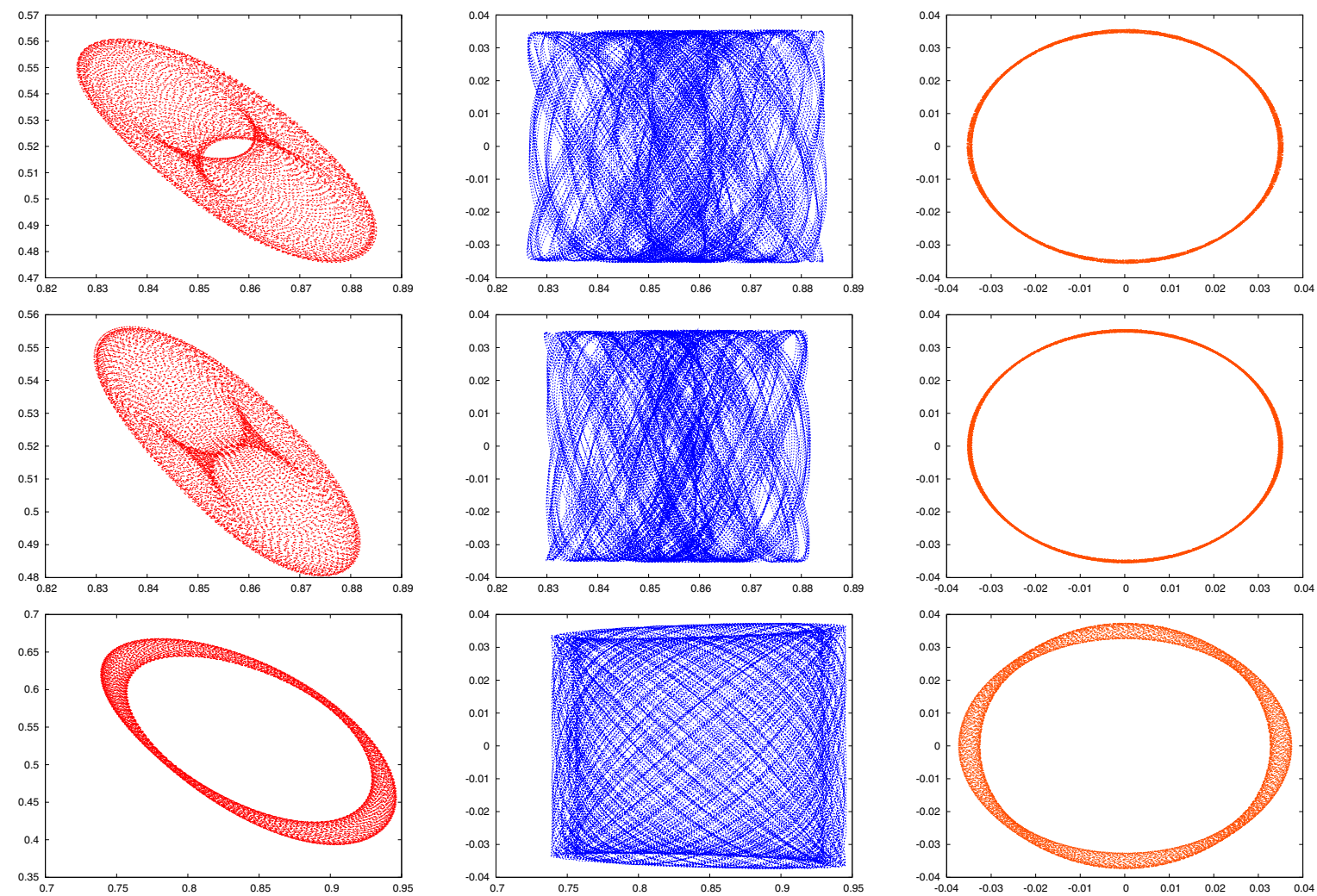

Figure 11. Invariant tori with initial conditions in the plane $I=2^{\circ}$. Left column: xy-projection. Center column: xz-projection. Right column: ż்-projection.

that the rigid-body effect of one of the primaries is to make this stability region smaller in both the equatorial plane of motion and in inclination. As an example, for $\nu \sim 10^{-3}$ (which is very close to the Sun-Jupiter mass parameter), the stability region in the RF3BP vanishes for inclinations $I \geq 10^{\circ}$, while in the Sun-Jupiter RTBP (or even in the real solar system) we know there are objects in stable orbits up to inclinations of $I \sim 40^{\circ}$ [9].

Dissipative mechanisms, such as solar radiation pressure, solar wind drag [42, 22], or the Poynting-Robertson effect, might destroy the stability regions as dissipation-induced instabilities do (see, for instance, [6]). In our case, though, we ignore the effect of solar radiation pressure on the orbiting spacecraft, an approximation that is excellent for a mission to a main-belt binary system. For near-Earth asteroid binary systems, a future study will be done to map out how this additional force modifies the dynamics.

Much work still needs to be done at a theoretical level before sending an actual probe to a binary asteroid. This paper contributes to this problem by applying to it the frequency analysis method and showing a way of finding stable satellite orbits. Future work following this line of investigation could consider several different aspects of the problem: first, one could compute and study similar stability regions for more complex rigid-body gravitational systems (like that in [47], for instance) or for two-body problems that are not in relative equilibrium (e.g., the rigid-body is rotating faster than the relative orbital velocity of the other primary 

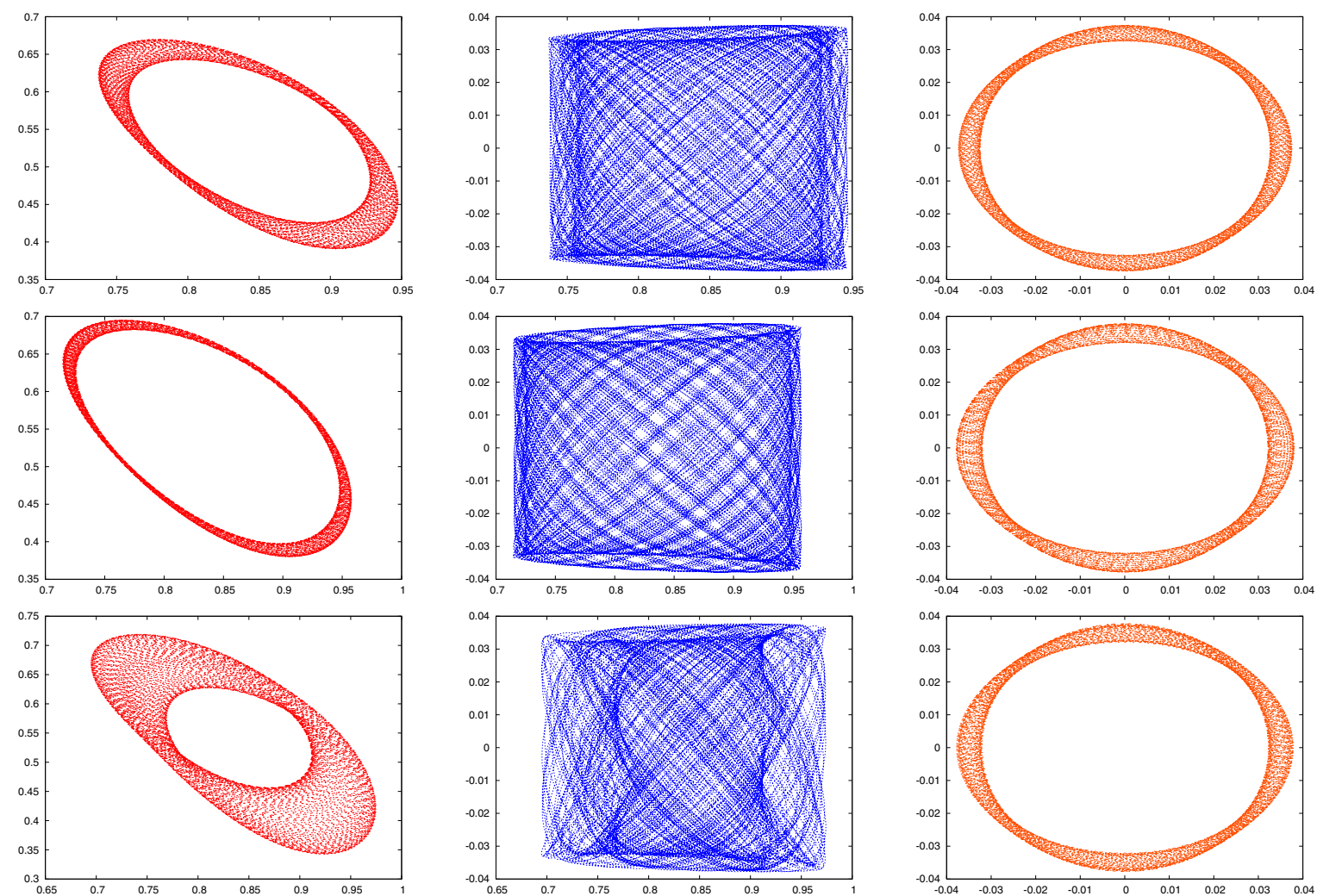

Figure 12. Invariant tori with initial conditions in the plane $I=2^{\circ}$. Left column: xy-projection. Center column: xz-projection. Right column: żं-projection.

[41]); second, it seems interesting to apply the same method for finding orbits that are not necessarily close to the equilibrium points (orbits that go around one of the primaries, encircle both primaries, retrograde orbits, etc.).

Appendix. Abelian reduction. General setting. In this appendix, we review the reduction process for a system that is invariant under the abelian Lie group $S O(2)$. We perform the reduction on both sides of the problem, Lagrangian and Hamiltonian, and show that they are equivalent via the reduced Legendre transform.

Let us start by assuming that the configuration space $Q$ can be written as a product of the circle $S^{1}$ and a manifold $B$ called shape space, i.e., $Q=S^{1} \times B$ and $q=\left(q^{0}, q^{\alpha}\right)=\left(\theta, r^{\alpha}\right)$, with $q \in Q, \theta \in S^{1}$ and $r^{\alpha} \in B \subseteq \mathbb{R}^{n}$.

Let us assume that the symmetry group $G=S O(2)=S^{1}$ acts trivially in the following way:

$$
\begin{aligned}
\Phi: G \times Q & \longrightarrow Q, \\
(\varphi, q) & \longmapsto \Phi_{\varphi}(q)=\Phi_{\varphi}\left(\theta, r^{\alpha}\right)=\left(\theta+\varphi, r^{\alpha}\right),
\end{aligned}
$$

where $G$ is a Lie group with Lie algebra $\mathfrak{g}=\mathbb{R}$ and dual Lie algebra $\mathfrak{g}^{*} \cong \mathbb{R}$. 

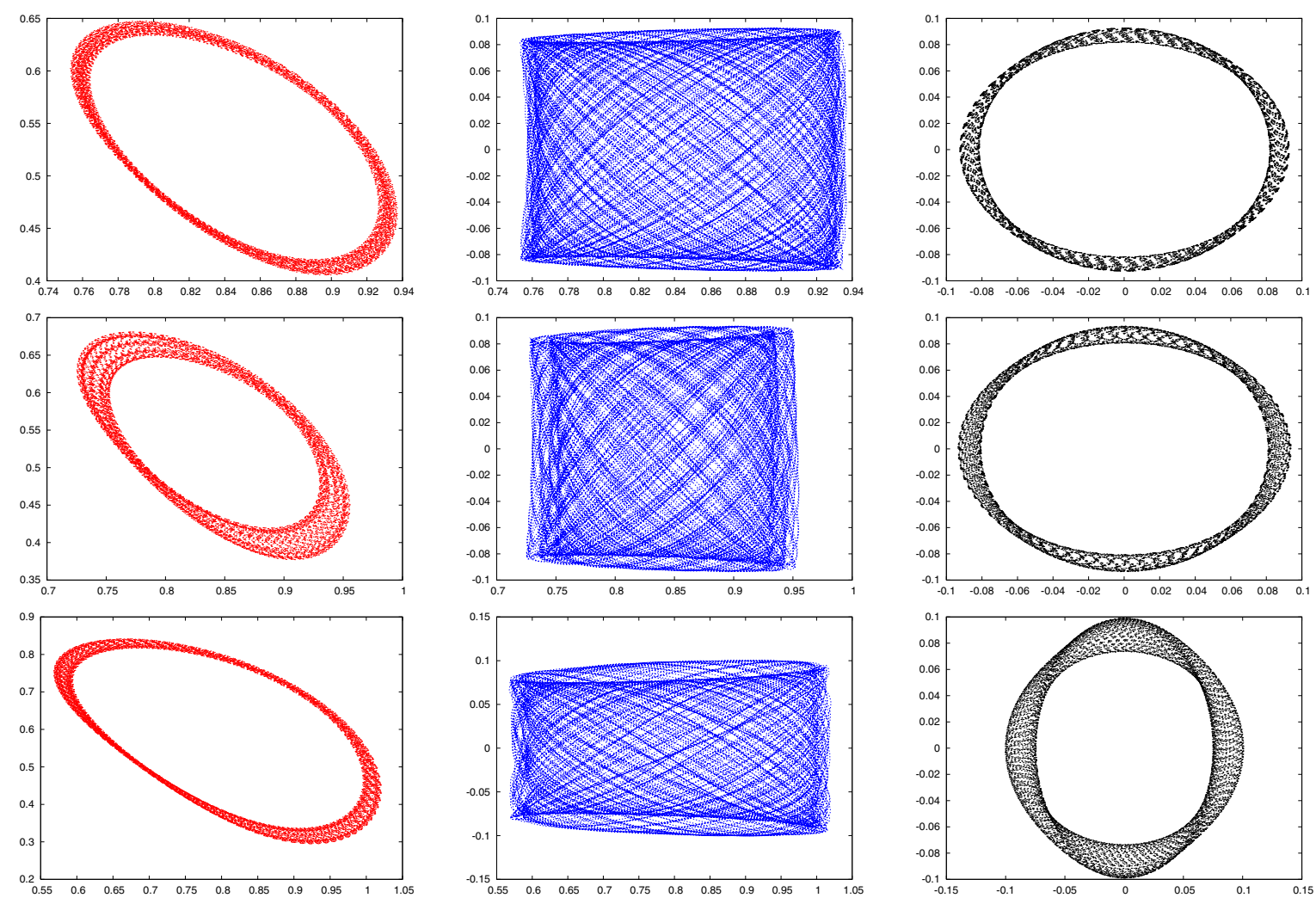

Figure 13. Invariant tori with initial conditions in the plane $I=5^{\circ}$. Left column: xy-projection. Center column: xz-projection. Right column: ż்-projection.

We also assume that the Lagrangian is of the type kinetic minus potential energy. Then, it can be written, in a local trivialization of the tangent bundle $T Q$, as follows:

$$
L(q, \dot{q})=K(q, \dot{q})-V(q)=\frac{1}{2} g_{i j} \dot{q}^{i} \dot{q}^{j}-V(q),
$$

where $g_{i j}$ is a Riemannian metric and summations over $i, j=0,1, \ldots, n$ are understood. The corresponding Hamiltonian on the cotangent bundle $T^{*} Q$ is given by

$$
H(q, p)=K(\mathbb{F} L(q, \dot{q}))+V(q)=\frac{1}{2} g^{i j} p_{i} p_{j}+V(q),
$$

where $g^{i j}$ is the inverse of the metric $g_{i j},(q, p)=\mathbb{F} L(q, \dot{q})$ is the Legendre transform of $(q, \dot{q})$ $\left(\left(q^{i}, p_{i}\right)=\left(q^{i}, g_{i j} \dot{q}^{j}\right)\right)$, and the symplectic form is canonical, i.e., $\Omega=d q^{i} \wedge d p_{i}$.

A.1. Lagrangian reduction. We start with the Lagrangian rewritten in the following form:

$$
L\left(r^{\alpha}, \dot{\theta}, \dot{r}^{\alpha}\right)=\frac{1}{2} g_{00} \dot{\theta}^{2}+g_{0 \alpha} \dot{\theta} \dot{r}^{\alpha}+\frac{1}{2} g_{\alpha \beta} \dot{r}^{\alpha} \dot{r}^{\beta}-V\left(r^{\alpha}\right),
$$

where $q^{0}=\theta \in \mathbb{T} ; q^{j}=r^{j}, j=1 \div n$; and $\alpha, \beta=1 \div n$. Note that $L \neq L(\theta)$. Thus, $\theta$ is a cyclic variable, and the corresponding conjugate momentum $p_{\theta}=g_{00} \dot{\theta}=\frac{\partial L}{\partial \dot{\theta}}$ is conserved. 

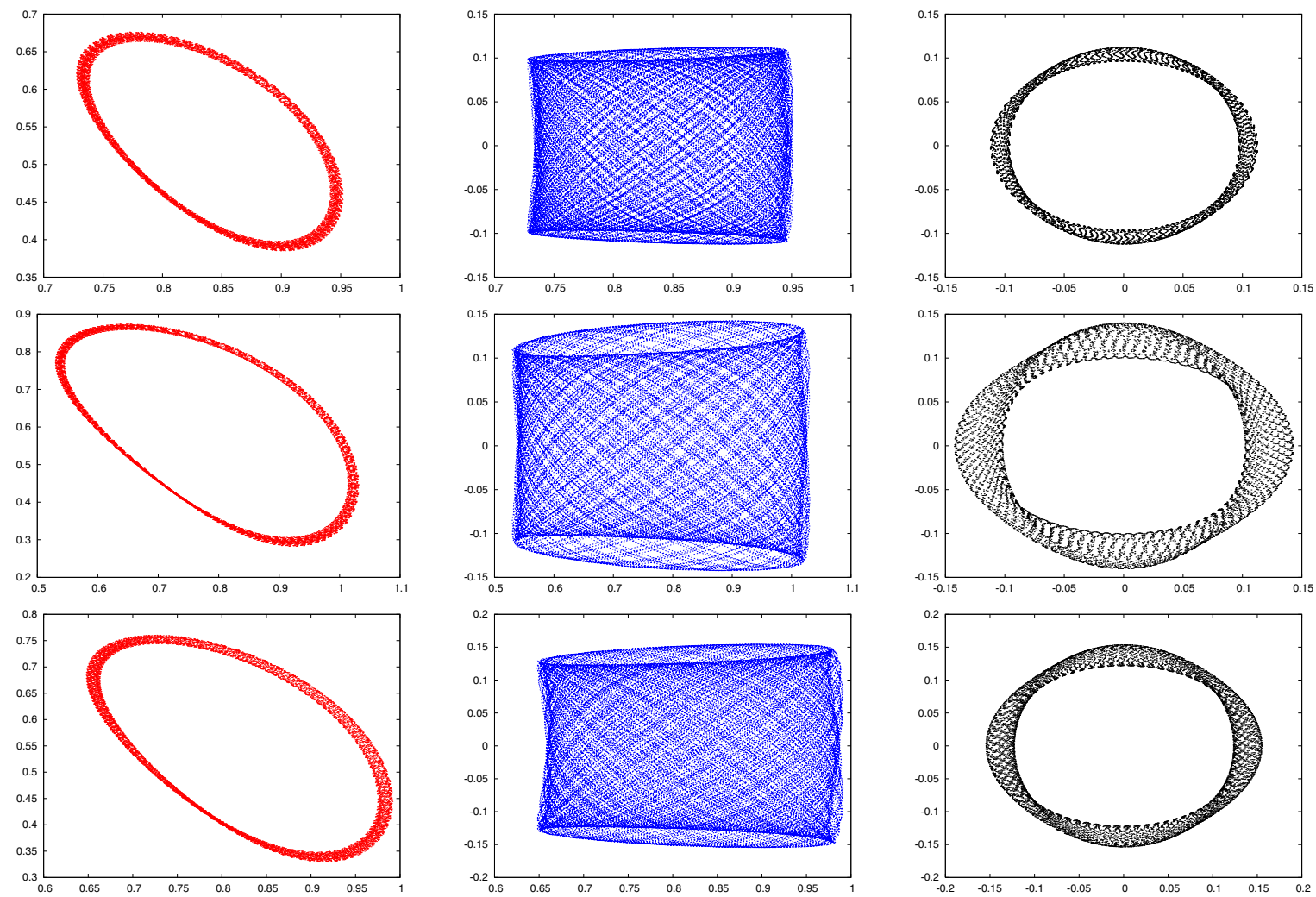

Figure 14. Invariant tori with initial conditions in the planes $I=6^{\circ}$ (first row), $I=7^{\circ}$ (second row), and $I=8^{\circ}$ (last row). Left column: xy-projection. Center column: xz-projection. Right column: żं-projection.

While the classical theory of Routh reduction is valid, we will use modern Routh reduction [30], which applies in a much more general framework.

The ingredients needed in the reduction process (see [30] for details) are the following:

- Infinitesimal generator: Given $\xi \in \mathfrak{g}^{*}$, the infinitesimal generator corresponding to the group action can be computed as follows:

$$
\xi_{Q}\left(\theta, r^{\alpha}\right)=\left.\frac{d}{d t}\left(\exp (t \xi) \cdot\left(\theta, r^{\alpha}\right)\right)\right|_{t=0}=\left(\left(\theta, r^{\alpha}\right),(\xi, 0)\right) .
$$

- Lagrangian momentum map: The associated momentum map $\mathbf{J}_{L}: T Q \rightarrow \mathfrak{g}^{*}$ is given by

$$
\mathbf{J}_{L}((q, \dot{q})) \cdot \xi_{Q}=\left\langle\mathbb{F} L(q, \dot{q}), \xi_{Q}(q)\right\rangle=\left\langle\left(g_{0 j} \dot{q}^{j}, g_{\alpha j} \dot{q}^{j}\right),(\xi, 0)\right\rangle=g_{0 j} \dot{q}^{j} \xi .
$$

Thus, $\mathbf{J}_{L}(q, \dot{q})=g_{00} \dot{\theta}+g_{0 \alpha} \dot{r}^{\alpha}$.

- Locked inertia tensor: The locked inertia tensor is the instantaneous tensor of inertia when the relative motion of the two bodies is locked. If we denote by $\langle\langle\cdot, \cdot\rangle\rangle$ the scalar product induced by the metric $g_{i j}$, the locked inertia tensor $\mathbb{I}\left(\theta, r^{\alpha}\right): \mathfrak{g} \longrightarrow \mathfrak{g}^{*}$ is given (locally) by

$$
\left\langle\mathbb{I}\left(\theta, r^{\alpha}\right) \eta, \xi\right\rangle=\left\langle\left\langle\left(\left(\theta, r^{\alpha}\right),(\eta, 0)\right),\left(\left(\theta, r^{\alpha}\right),(\xi, 0)\right)\right\rangle\right\rangle=g_{00} \eta \xi .
$$



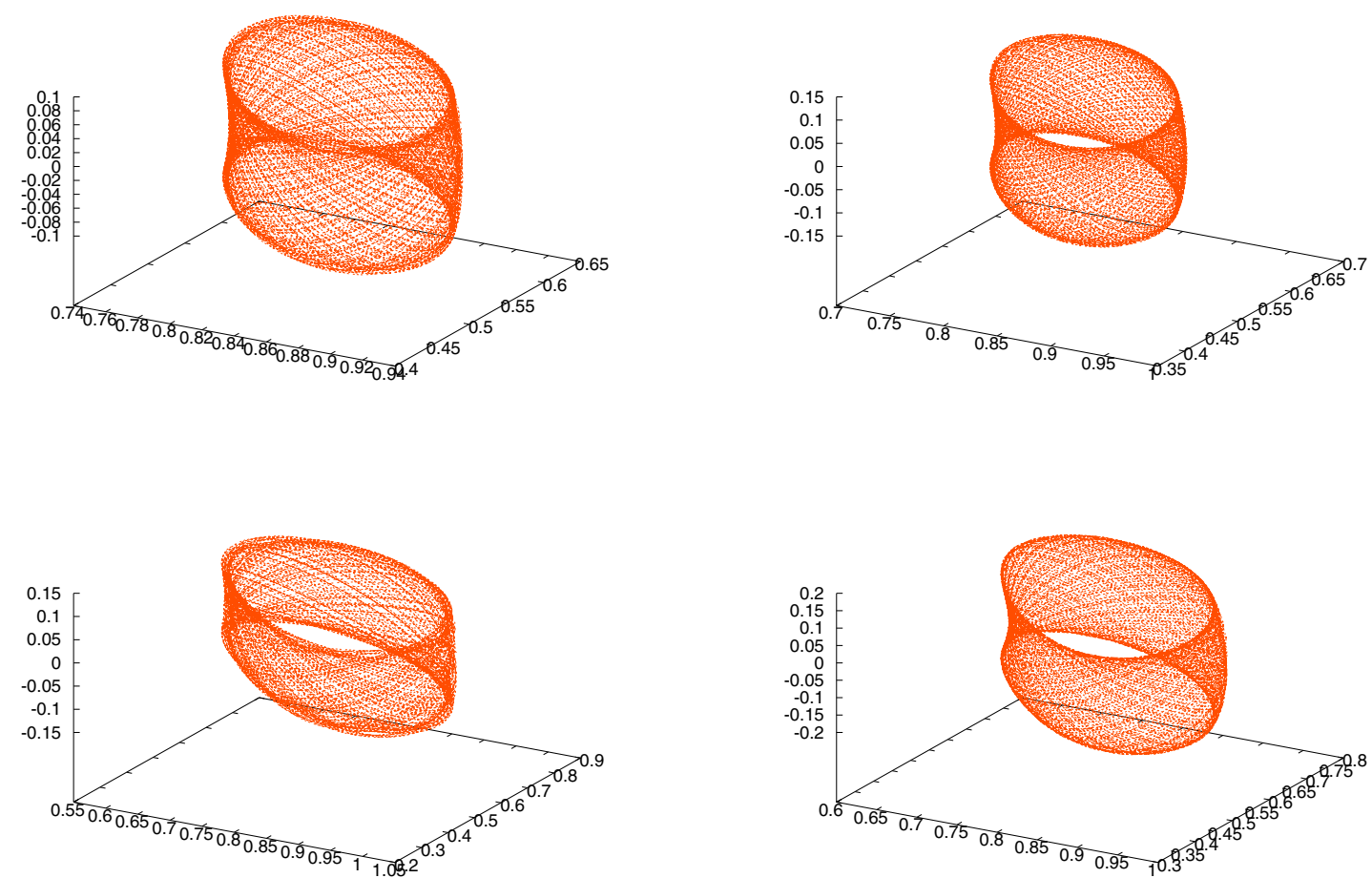

Figure 15. Left column: xyz-projections of two different invariant tori with initial conditions in the plane $I=5^{\circ}$. Right column: Invariant tori with initial conditions in the planes $I=6^{\circ}$ (top) and $I=8^{\circ}$ (bottom).

Then, $\mathbb{I}\left(\theta, r^{\alpha}\right)=g_{00}\left(r^{\alpha}\right)$.

- Mechanical connection: The connection $\mathcal{A}: T Q \longrightarrow \mathfrak{g}$ can be written (locally) as $\mathcal{A}\left(\theta, r^{\alpha}\right)\left(\theta, r^{\alpha}, \dot{\theta}, \dot{r}^{\alpha}\right)=\mathbb{I}^{-1} \mathbf{J}\left(\mathbb{F} L\left(\theta, r^{\alpha}, \dot{\theta}, \dot{r}^{\alpha}\right)\right)=g_{00}^{-1} g_{0 j} \dot{q}^{j}$. Thus,

$$
\mathcal{A}\left(\theta, r^{\alpha}\right)\left(\theta, r^{\alpha}, \dot{\theta}, \dot{r}^{\alpha}\right)=\dot{\theta}+g_{00}^{-1} g_{0 \alpha} \dot{r}^{\alpha} .
$$

From $\mathcal{A}$, we can obtain the related one-form: $\mathcal{A}\left(\theta, r^{\alpha}\right)=d \theta+A_{\alpha} d r^{\alpha}$, where $A_{\alpha}=$ $g_{00}^{-1} g_{0 \alpha}$, and the curvature $\mathcal{B}=d \mathcal{A}=B_{\alpha \beta} d r^{\alpha} \wedge d r^{\beta}$ has components given (locally) by $B_{\alpha \beta}=\left(\frac{\partial A_{\alpha}}{\partial r^{\beta}}-\frac{\partial A_{\beta}}{\partial r^{\alpha}}\right)$. For a given $\mu \in \mathfrak{g}^{*} \cong \mathbb{R}$, the mechanical connection on the fiber $Q \rightarrow Q / G$ is

$$
\alpha_{\mu}\left(\theta, r^{\alpha}\right)=\mu d \theta+\mu A_{\alpha} d r^{\alpha} .
$$

- Amended potential: For $\mu \in \mathfrak{g}^{*}$, the amended potential is defined as

$$
V_{\mu}(q)=V(q)+\frac{1}{2}\left\langle\mu, \mathbb{I}^{-1}(q) \mu\right\rangle=V(q)+\frac{1}{2} g_{00}^{-1} \mu^{2} .
$$

- Routhian: The Routhian is a function on $T Q$ defined as

$$
R^{\mu}=\frac{1}{2}\|\operatorname{Hor}(q, \dot{q})\|^{2}-V_{\mu},
$$


where $\operatorname{Hor}(q, \dot{q})=\left(-g_{00}^{-1} g_{0 \alpha} \dot{r}^{\alpha}, \dot{r}^{\alpha}\right)$ is the horizontal component of $(q, \dot{q})$ and the norm is given by the $g_{i j}$ metric. Then, locally, we can write

$$
R^{\mu}=\frac{1}{2}\left(g_{\alpha \beta}-g_{00}^{-1} g_{0 \alpha} g_{0 \beta}\right) \dot{r}^{\alpha} \dot{r}^{\beta}-\frac{1}{2} g_{00}^{-1} \mu^{2}-V\left(r^{\alpha}\right) .
$$

The general reduction theory $[28,30]$ tells us that if a curve $q(t)$ in $Q$ satisfying $\mathbf{J}_{L}(q, \dot{q})=\mu$ is a solution of the Euler-Lagrange equations for the Lagrangian $L(q, \dot{q})$, then the induced curve on $Q / G_{\mu}$ satisfies the reduced Lagrangian variational principle; that is, the variational principle of Lagrange and d'Alembert on $Q / G_{\mu}$ with magnetic term $\mathcal{B}$ and the Routhian dropped to $T\left(Q / G_{\mu}\right)$.

Let be $\hat{R}^{\mu}$ the reduced Routhian, that is, the Routhian (A.1) dropped to the reduced space $\mathbf{J}_{L}^{-1}(\mu) / S^{1}$. Then (locally),

$$
\hat{R}^{\mu}=\frac{1}{2} h_{\alpha \beta} \dot{r}^{\alpha} \dot{r}^{\beta}-V_{\mu}\left(r^{\alpha}\right)
$$

where $h_{\alpha \beta}=g_{\alpha \beta}-g_{00}^{-1} g_{0 \alpha} g_{0 \beta}$ is a metric in the reduced space and $V_{\mu}\left(r^{\alpha}\right)$ is the amended potential.

- Equations of Lagrange-Routh: The equations of motion in the reduced space $\mathbf{J}_{L}^{-1}(\mu) / S^{1}$ are given by

$$
\frac{d}{d t} \frac{\partial \hat{R}^{\mu}}{\partial \dot{r}^{\alpha}}-\frac{\partial \hat{R}^{\mu}}{\partial r^{\alpha}}=-\mu B_{\alpha \beta} \dot{r}^{\beta}
$$

where $B_{\alpha \beta}=\frac{\partial A_{\alpha}}{\partial r^{\beta}}-\frac{\partial A_{\beta}}{\partial r^{\alpha}}$ and $A_{\alpha}=g_{00}^{-1} g_{0 \alpha}$. More concretely,

$$
h_{\alpha \beta} \ddot{r}^{\beta}+\left(\frac{\partial h_{\alpha \beta}}{\partial r^{\gamma}}-\frac{1}{2} \frac{\partial h_{\beta \gamma}}{\partial r^{\alpha}}\right) \dot{r}^{\beta} \dot{r}^{\gamma}=-\frac{\partial V_{\mu}\left(r^{\alpha}\right)}{\partial r^{\alpha}}-\mu B_{\alpha \beta} \dot{r}^{\beta} .
$$

A.2. Cotangent bundle reduction. Now, we perform the corresponding reduction in the Hamiltonian side [27]. Let us consider that the initial Hamiltonian can be written (locally) as

$$
H\left(r^{\alpha}, p_{\theta}, p_{r}^{\alpha}\right)=\frac{1}{2} g^{00} p_{\theta}^{2}+g^{0 \alpha} p_{\theta} p_{\alpha}+\frac{1}{2} g^{\alpha \beta} p_{\alpha} p_{\beta}+V\left(r^{\alpha}\right),
$$

where the metric elements $g^{i j}$ correspond to the inverse of the metric $g_{i j}$. That is, $g_{i j} g^{j k}=\delta_{i}^{k}$, where $i, j, k=0, \ldots, n$, and $\delta_{i}^{k}$ denotes the Kronecker delta function.

Thus, we assume that the initial Hamiltonian is invariant under the action of the abelian symmetry group $G=S O(2)=S^{1}$.

Let us perform the computations of all the extra ingredients needed in the reduction in the Hamiltonian side [27], as follows:

- Momentum map: The momentum map corresponds to the angular momentum of the system: $\mathbf{J}: T^{*} Q \longrightarrow \mathfrak{g}^{*}$

$$
\left\langle\mathbf{J}\left(\theta, r^{\alpha}, p_{\theta}, p_{r^{\alpha}}\right), \xi\right\rangle=\left\langle\left(p_{\theta}, p_{\alpha}\right),(\xi, 0)\right\rangle=p_{\theta} \xi .
$$

Thus, $\mathbf{J}\left(\theta, r^{\alpha}, p_{\theta}, p_{r^{\alpha}}\right)=p_{\theta}$. 
- Momentum shift: In this case, it is convenient to perform a shift of the momenta from $\mathbf{J}^{-1}(\mu)$ to $\mathbf{J}^{-1}(0)$, and also in the corresponding reduced spaces, in the following way:

$$
\begin{array}{rcc}
\mathbf{J}^{-1}(\mu)=\left\{\left(\theta, r^{\alpha}, \mu, p_{\alpha}\right)\right\} & \stackrel{t^{\mu}}{\longrightarrow} & \mathbf{J}^{-1}(0)=\left\{\left(\theta, r^{\alpha}, 0, \tilde{p}_{\alpha}\right)\right\}, \\
\downarrow & \downarrow \\
\mathbf{J}^{-1}(\mu) / G_{\mu}=\mathbf{J}^{-1}(\mu) / S^{1} \stackrel{t_{G}^{\mu}}{\longrightarrow} & \mathbf{J}^{-1}(0) / S^{1}=\mathbf{J}^{-1}(0) / G,
\end{array}
$$

where

$$
\begin{aligned}
t^{\mu}\left(\theta, r^{\alpha}, \mu, p_{\alpha}\right) & =\left(\theta, r^{\alpha}, \mu, p_{\alpha}\right)-\left(\theta, r^{\alpha}, \mu, \mu A_{\alpha}\right) \\
& =\left(\theta, r^{\alpha}, 0, p_{\alpha}-\mu A_{\alpha}\right)=\left(\theta, r^{\alpha}, \tilde{p}_{\theta}, \tilde{p}_{\alpha}\right)
\end{aligned}
$$

Thus, the shifting map is given by $\tilde{p}_{\alpha}=p_{\alpha}-\mu A_{\alpha}$ and $\tilde{p}_{\theta}=0$.

- Reduced Hamiltonian: In $\mathbf{J}^{-1}(0) / G$ we have $H_{\alpha_{\mu}}=\frac{1}{2}\|\tilde{p}\|^{2}+V_{\mu}$, where $\|\cdot\|$ is the norm related to the $g^{i j}$ metric and $V_{\mu}=V+\frac{1}{2} \mathbb{I}^{-1} \mu^{2}$ is the amended potential. Thus, recalling that $\tilde{p}_{\theta}=0$, the Hamiltonian in the reduced space $\mathbf{J}^{-1}(0) / G$ is

$$
H_{\mu}\left(r^{\alpha}, p_{\alpha}\right)=\frac{1}{2} g^{\alpha \beta} \tilde{p}_{\alpha} \tilde{p}_{\beta}+V\left(r^{\alpha}\right)+\frac{1}{2} \mu^{2} g_{00}^{-1} \quad \text { for } \alpha, \beta=1 \div n \text {. }
$$

- Reduced symplectic form: In general, in the reduced space, the symplectic form is not canonical. The projection is given by the map

$$
\left(\left(T^{*} Q\right)_{\mu}, \Omega_{\mu}\right) \stackrel{P_{\mu}}{\longrightarrow}\left(\left(T^{*}(Q / G), \omega-B_{\mu}\right),\right.
$$

where the "reduced" symplectic form is

$$
\omega_{\mu}=\omega-B_{\mu}=d r^{\alpha} \wedge d \tilde{p}_{\alpha}-\mu \frac{\partial A_{\alpha}}{\partial r^{\beta}} d r^{\beta} \wedge d r^{\alpha} .
$$

- Hamiltonian equations: The Hamiltonian equations are given by [28]

$$
\mathbf{i}_{\left(\dot{r}^{\alpha} \partial_{r} \alpha+\dot{\tilde{p}}_{\alpha} \partial_{\tilde{p}_{\alpha}}\right)} \omega_{\mu}=d H_{\mu}
$$

where $\mathbf{i}_{X} \Omega$ denotes the interior product (or contraction) of the vector field $X$ and the 1 -form $\Omega$. Computing both sides of this identity,

$$
\begin{aligned}
\mathbf{i}_{\left(\dot{r}^{\alpha} \partial_{r^{\alpha}}+\dot{\tilde{p}}_{\alpha} \partial_{\tilde{p}_{\alpha}}\right)} \omega_{\mu} & =\dot{r}^{\alpha} d \tilde{p}_{\alpha}-\mu \frac{\partial A_{\alpha}}{\partial r^{\beta}} \dot{r}^{\beta} d r^{\alpha}+\mu \frac{\partial A_{\alpha}}{\partial r^{\beta}} \dot{r}^{\alpha} d r^{\beta}-\dot{\tilde{p}}_{\alpha} d r^{\alpha}, \\
d H_{\mu} & =\frac{\partial H_{\mu}}{\partial r^{\alpha}} d r^{\alpha}+\frac{\partial H_{\mu}}{\partial \tilde{p}_{\alpha}} d \tilde{p}_{\alpha},
\end{aligned}
$$

we obtain the equations of motion in the reduced $J^{-1}(0) / G$ space:

$$
\dot{r}^{\alpha}=\frac{\partial H_{\mu}}{\partial \tilde{p}_{\alpha}}, \quad \dot{\tilde{p}}_{\alpha}=-\frac{\partial H_{\mu}}{\partial r^{\alpha}}-\mu\left(\frac{\partial A_{\alpha}}{\partial r^{\beta}}-\frac{\partial A_{\alpha}}{\partial r^{\beta}}\right) \dot{r}^{\beta} .
$$

Finally, we can write them more explicitly as

$$
\dot{r}^{\alpha}=g^{\alpha \beta} \tilde{p}_{\beta}, \quad \dot{\tilde{p}}_{\alpha}=-\frac{1}{2} \frac{\partial g^{\beta \gamma}}{\partial r^{\alpha}} \tilde{p}_{\beta} \tilde{p}_{\gamma}-\frac{\partial V\left(r^{\alpha}\right)}{\partial r^{\alpha}}-\frac{1}{2} \mu^{2} \frac{\partial g_{00}^{-1}}{\partial r^{\alpha}}-\mu B_{\alpha \beta} g^{\beta \gamma} \tilde{p}_{\gamma} .
$$


A.3. Reduced Legendre transform. The correspondence between the reduced equations of motion on the Hamiltonian and Lagrangian sides is given by the reduced Legendre transform. We start with the reduced Routhian (A.2),

$$
\hat{R}^{\mu}=\frac{1}{2}\left(g_{\alpha \beta}-g_{00}^{-1} g_{0 \alpha} g_{0 \beta}\right) \dot{r}^{\alpha} \dot{r}^{\beta}-\frac{1}{2} g_{00}^{-1} \mu^{2}-V\left(r^{\alpha}\right),
$$

and the shifted momenta,

$$
\tilde{p}_{\alpha}=\frac{\partial \hat{R}^{\mu}}{\partial \dot{r}^{\alpha}}=\left(g_{\alpha \beta}-g_{00}^{-1} g_{0 \alpha} g_{0 \beta}\right) \dot{r}^{\beta} .
$$

Using the identities

$$
\begin{aligned}
g_{0 \alpha} g^{0 \beta}+g_{\alpha \gamma} g^{\gamma \beta} & =\delta_{\alpha}^{\beta}, \\
g^{\alpha \beta} g_{\beta 0}+g^{\alpha 0} g_{00} & =0,
\end{aligned}
$$

we obtain the first equation in (A.4): $g^{\alpha \beta} \tilde{p}_{\beta}=\dot{r}^{\alpha}$.

Now, in order to recover the reduced Lagrange-Routh equations (A.3), we compute the time-derivative of the shifted momenta

$$
\dot{\tilde{p}}_{\alpha}=\left(g_{\alpha \beta}-g_{00}^{-1} g_{0 \alpha} g_{0 \beta}\right) \ddot{r}^{\beta}+\frac{\partial}{\partial r^{\gamma}}\left(g_{\alpha \beta}-g_{00}^{-1} g_{0 \alpha} g_{0 \beta}\right) \dot{r}^{\gamma} \dot{r}^{\beta},
$$

and the derivative with respect to $r^{\alpha}$ of the identities (A.5),

$$
\begin{gathered}
\frac{\partial g_{0 \epsilon}}{\partial r^{\alpha}} g^{0 \beta}+g_{0 \epsilon} \frac{\partial g^{0 \beta}}{\partial r^{\alpha}}+\frac{\partial g_{\epsilon \gamma}}{\partial r^{\alpha}} g^{\gamma \beta}+g_{\epsilon \gamma} \frac{\partial g^{\gamma \beta}}{\partial r^{\alpha}}=0 \\
\frac{\partial g^{\beta \gamma}}{\partial r^{\alpha}} g_{\gamma 0}+g^{\beta \gamma} \frac{\partial g_{\gamma 0}}{\partial r^{\alpha}}+\frac{\partial g^{\beta 0}}{\partial r^{\alpha}} g_{00}+g^{\beta 0} \frac{\partial g_{00}}{\partial r^{\alpha}}=0 .
\end{gathered}
$$

If we substitute the last three identities together with (A.5) into the second equation of (A.4), we obtain

$$
\begin{array}{r}
\left(g_{\alpha \beta}-g_{00}^{-1} g_{0 \alpha} g_{0 \beta}\right) \ddot{r}^{\beta}+\frac{\partial}{\partial r^{\gamma}}\left(g_{\alpha \beta}-g_{00}^{-1} g_{0 \alpha} g_{0 \beta}\right) \dot{r}^{\gamma} \dot{r}^{\beta} \\
=\frac{1}{2} \frac{\partial}{\partial r^{\alpha}}\left(g_{\beta \gamma}-g_{00}^{-1} g_{0 \beta} g_{0 \gamma}\right) \dot{r}^{\beta} \dot{r}^{\gamma}-\frac{\partial V\left(r^{\alpha}\right)}{\partial r^{\alpha}}-\frac{1}{2} \mu^{2} \frac{\partial g_{00}^{-1}}{\partial r^{\alpha}}-\mu B_{\alpha \beta} \dot{r}^{\beta}
\end{array}
$$

which exactly corresponds to the Lagrange-Routh equations (A.3).

Acknowledgment. The authors thank J. M. Mondelo for providing them with a program for frequency analysis.

\section{REFERENCES}

[1] V. I. Arnold, Proof of A.N. Kolmogorov's theorem on the preservation of quasi-periodic motions under small perturbations of the Hamiltonian, Russian Math. Surveys, 18 (1963), pp. 9-36.

[2] J. Bellerose And D. J. Scheeres, Stability of Equilibrium Points in the Restricted Full Three Body Problem, preprint, University of Michigan, Ann Arbor, MI, 2006; available online at http://www-personal.engin.umich.edu/ scheeres/reprints.html. 
[3] A. M. Bloch, P. S. Krishnaprasad, J. E. Marsden, and T. S. Ratiu, Dissipation induced instabilities, Ann. Inst. H. Poincaré Anal. Non Linéaire, 11 (1994), pp. 37-90.

[4] B. C. Carlson, Computing elliptic integrals by duplication, Numer. Math., 33 (1979), pp. 1-16.

[5] H. Cendra and J. E. Marsden, Geometric mechanics and the dynamics of asteroid pairs, Dyn. Syst., 20 (2005), pp. 3-21.

[6] M. G. Clerc and J. E. Marsden, Dissipation-induced instabilities in an optical cavity laser: A mechanical analog near the 1: 1 resonance, Phys. Rev. E, 64 (2001), paper 067603.

[7] F. Gabern, On the Dynamics of the Trojan Asteroids, Ph.D. thesis, Department of Applied Mathematics and Analysis, University of Barcelona, Barcelona, Spain, 2003; available online at http://www.maia. ub.es/dsg/2003/.

[8] F. Gabern And À. JorbA, A restricted four-body model for the dynamics near the Lagrangian points of the Sun-Jupiter system, Discrete Contin. Dyn. Syst. Ser. B, 1 (2001), pp. 143-182.

[9] F. Gabern, A. Jorba, And U. LocAtelli, On the construction of the Kolmogorov normal form for the Trojan asteroids, Nonlinearity, 18 (2005), pp. 1705-1734.

[10] F. Gabern, À. Jorba, And P. Robutel, On the accuracy of restricted three-body models for the Trojan motion, Discrete Contin. Dynam. Systems, 11 (2004), pp. 843-854.

[11] F. Gabern, W. S. Koon, And J. E. Mardden, Spacecraft dynamics near a binary asteroid, Discrete Contin. Dynam. Systems, supplement (2005), pp. 297-306.

[12] F. Gabern, W. S. Koon, And J. E. Marsden, Parking a spacecraft near an asteroid pair, J. Guid. Control Dyn., to appear.

[13] A. Giongilli And C. Skokos, On the stability of the Trojan asteroids, Astron. Astrophys., 317 (1997), pp. $254-261$.

[14] G. Gómez, À. Jorba, J. Masdemont, And C. Simó, Dynamics and Mission Design near Libration Points, Vol. IV, World Sci. Monogr. Ser. Math. 5, World Scientific Publishing, River Edge, NJ, 2001.

[15] G. Gómez, W. S. Koon, M. Lo, J. E. Marsden, J. Masdemont, and S. D. Ross, Connecting orbits and invariant manifolds in the spatial restricted three-body problem, Nonlinearity, 17 (2004), pp. $1571-1606$.

[16] G. Gómez, J. M. Mondelo, and C. Simó, Refined Fourier Analysis: Procedures, Error Estimates and Applications, preprint, University of Barcelona, Barcelona, Spain, 2001; available online at http://www.maia.ub.es/dsg/2001/.

[17] W. R. Johnston, Asteroids with Satellites, available online at Johnston's Archive: http://www. johnstonsarchive.net/astro/asteroidmoons.html (last visit in May 2005).

[18] À. Jorba And J. Masdemont, Dynamics in the centre manifold of the collinear points of the restricted three body problem, Phys. D, 132 (1999), pp. 189-213.

[19] A. N. Kolmogorov, On the persistence of conditionally periodic motions under a small change of the Hamilton function, Dokl. Akad. Nauk SSSR, 98 (1954), pp. 527-530.

[20] W. S. Koon, J. E. Marsden, S. Ross, M. Lo, and D. J. Scheeres, Geometric mechanics and the dynamics of asteroid pairs, Ann. New York Acad. Sci., 1017 (2004), pp. 11-38.

[21] J. LASKAR, Introduction to frequency map analysis, in Hamiltonian Systems with Three or More Degrees of Freedom, NATO Adv. Sci. Inst. Ser. C Math. Phys. Sci. 533, C. Simó, ed., Kluwer Academic Publishers, Dordrecht, The Netherlands, 1999, pp. 134-150.

[22] J. C. Liou AND H. A. ZooK, An asteroidal dust ring of micron-sized particles trapped in $1: 1$ mean motion with Jupiter, Icarus, 113 (1995), pp. 403-414.

[23] R. DE LA Llave, A tutorial on KAM theory, in Smooth Ergodic Theory and Its Applications, Proc. Sympos. Pure Math. 69, AMS, Providence, RI, 2001, pp. 175-292.

[24] A. J. Maciejewski, Reduction, relative equilibria and potential in the two rigid bodies problem, Celest. Mech. Dyn. Astron., 63 (1996), pp. 1-28.

[25] W. D. MacMillan, The Theory of the Potential, McGraw-Hill, New York, 1930.

[26] J. L. Margot, M. C. Nolan, L. A. M. Benner, S. J. Ostro, R. F. Jurgens, J. D. Giorgini, M. A. Slade, And D. B. CAmpBell, Binary asteroids in the near-Earth object population, Science, 296 (2002), pp. 1445-1448.

[27] J. E. Marsden, Lectures on Mechanics, London Math. Soc. Lecture Note Ser. 174, Cambridge University Press, Cambridge, UK, 1992.

[28] J. E. Marsden And T. S. Ratiu, Introduction to Mechanics and Symmetry, Texts in Appl. Math. 17, Springer-Verlag, New York, 1999. 
[29] J. E. Marsden And S. D. Ross, New methods in celestial mechanics and mission design, Bull. Amer. Math. Soc. (N.S.), 43 (2006), pp. 43-73.

[30] J. E. Marsden, T. S. Ratiu, And J. Scheurle, Reduction theory and the Lagrange-Routh equations, J. Math. Phys., 41 (2000), pp. 3379-3429.

[31] J. E. Marsden and J. Scheurle, Lagrangian reduction and the double spherical pendulum, Z. Angew. Math. Phys., 44 (1993), pp. 17-43.

[32] R. McKenzie and V. Szebehely, Nonlinear stability around the triangular libration points, Celest. Mech. Dyn. Astron., 23 (1981), pp. 223-229.

[33] W. J. Merline, S. J. Weidenschilling, D. D. Durda, J. L. Margot, P. Pravec, and A. D. Storrs, Asteroids do have satellites, in Asteroids III, W. F. Bottke, A. Cellino, P. Paolicchi, and R. P. Binzel, eds., University of Arizona Press, Tucson, AZ, 2002, pp. 289-312.

[34] J. Moser, On invariant curves of area-preserving mappings of an annulus, Nachr. Akad. Wiss. Göttingen Math.-Phys. Kl. II, 2 (1962), pp. 1-20.

[35] Y. G. Он, A stability criterion for Hamiltonian systems with symmetry, J. Geom. Phys., 4 (1987), pp. $163-182$.

[36] P. Robutel, F. Gabern, And À. Jorba, The observed Trojans and the global dynamics around the Lagrangian points of the Sun-Jupiter system, Celest. Mech. Dyn. Astron., 92 (2005), pp. 53-69.

[37] P. Robutel AND J. LASKAR, Frequency map and global dynamics in the Solar System I, Icarus, 152 (2001), pp. 4-28.

[38] D. J. ScheEREs, Dynamics about uniformly rotating triaxial ellipsoids: Applications to asteroids, Icarus, 110 (1994), pp. 225-238.

[39] D. J. Scheeres, Stability in the full two-body problem, Celest. Mech. Dyn. Astron., 83 (2002), pp. 155169.

[40] D. J. Scheeres, Stability of relative equilibria in the full two-body problem, Ann. New York Acad. Sci., 1017 (2004), pp. 81-94.

[41] D. J. Scheeres And J. Bellerose, The restricted Hill full 4-body problem: Application to spacecraft motion about binary asteroids, Dyn. Syst., 20 (2005), pp. 23-44.

[42] D. W. Schuerman, The restricted three-body problem including radiation pressure, Astrophys. J., 238 (1980), pp. 337-342.

[43] M. B. Sevryuk, The classical KAM theory at the dawn of the twenty-first century, Moscow Math. J., 3 (2003), pp. 1113-1144.

[44] J. C. Simo, D. Lewis, And J. E. Marsden, Stability of relative equilibria. I. The reduced energymomentum method, Arch. Rational Mech. Anal., 115 (1991), pp. 15-59.

[45] V. Szebehely, Theory of Orbits, Academic Press, New York, 1967.

[46] L. V. Vela-Arevalo And J. E. Marsden, Time-frequency analysis of the restricted three-body problem: Transport and resonance transitions, Classical Quantum Gravity, 21 (2004), pp. S351-S375.

[47] R. A. Werner And D. J. Scheeres, Exterior gravitation of a polyhedron derived and compared with harmonic and mascon gravitation representations of asteroid 4769 Castalia, Celest. Mech. Dyn. Astron., 65 (1997), pp. 313-344. 